\title{
Computational and experimental designing of imprinted sorbent for the determination of nitroxidative stress products: an analysis of 4- hydroxyphenylacetic acid conversion
}

\author{
Marta Janczura ${ }^{1}$, Monika Sobiech ${ }^{1}$, Joanna Giebułtowicz ${ }^{2}$, and Piotr Luliński ${ }^{1, \star}$ (1) \\ ${ }^{1}$ Department of Organic Chemistry, Faculty of Pharmacy, Medical University of Warsaw, Banacha 1, 02-097 Warsaw, Poland \\ ${ }^{2}$ Department of Bioanalysis and Drugs Analysis, Faculty of Pharmacy, Medical University of Warsaw, Banacha 1, 02-097 Warsaw, \\ Poland
}

Received: 3 November 2020 Accepted: 27 January 2021 Published online:

11 February 2021

(C) The Author(s) 2021

\begin{abstract}
It this paper, the comprehensive design process was carried out to fabricate selective, molecularly imprinted polymer (MIP). The material was used as a sorbent in the optimized analytical method, aimed at verifying the hypothesis that the conditions of an analytical process could convert 4-hydroxyphenylacetic acid to 4-hydro-3-nitrophenylacetic acid, comprising a series of novel studies. The design stage consisted of the analysis of specificity of the MIP, synthesized from the cross-linker and used five various functional monomers independently, in the presence of four different templates. The MIP from 1-vinylimidazole, imprinted by 4-hydroxyphenylacetic acid, revealed the highest specificity in relation to 4-hydro-3-nitrophenylacetic acid, with an affinity factor equal to 3 , and the highest selectivity from a group of structurally similar and biologically important biomolecules. The theoretical analysis revealed that electrostatic interaction between the analyte and the polymer matrix enhanced selectivity. The physicochemical characterization showed the specific surface area of the MIP as being equal to $368.6 \mathrm{~m}^{2} \mathrm{~g}^{-1}$, and the presence of nitrogen atoms at the level of $6.80 \%$ wt., confirming the monomer residue in the material structure. The MIP was applied in the solid phase extraction protocol, allowing for the analysis of 4-hydroxy-3-nitrophenylacetic acid in a human urine sample. Finally, the conversion of 4-hydroxyphenylacetic acid in human urine in nitrate and nitrite salts at low $\mathrm{pH}$ conditions revealed an almost twofold increase in 4-hydro-3-nitrophenylacetic acid to $775 \pm 81 \mathrm{ng} \mathrm{L}^{-1}$. The results also confirmed
\end{abstract}

Handling Editor: Yaroslava Yingling.

Address correspondence to E-mail: piotr.lulinski@wum.edu.pl 
the applicability of the new MIP sorbent for the purpose of analysis of low levels of analyte, present in the complex sample.

\section{Introduction}

Advanced materials are in high demand in the field of analytical chemistry, as they could improve the characterization of analytical methods, viz. they allow for lower limits of quantification (LLOQ), improve accuracy, enhance selectivity and minimize matrix effects. Among the various materials, the polymeric sorbents, with their predetermined capabilities, are considered the most attractive. Here, molecularly imprinted polymers (MIPs) are recognized as a valuable class of materials, characterized by prearranged properties, due to the three-dimensional cavities formed in the polymer network by the template-tailored synthesis [1]. One of the main attributes of imprinted materials is high specificity, derived from the spatial modification of the polymer surface by the template, an advantage that has paved the way for widespread application of MIPs in different kinds of sensors, as recognition elements [2-4] in synthesis and catalysis [5, 6] and as potential drug delivery devices [7-9]. Nevertheless, MIPs are predominantly known as highly useful materials for separation purposes, due to their capability to clean up complex samples [10-13]. However, the adsorption on the resulting MIPs is governed by different factors, making the process less predictable and often unsatisfactory. For that reason, the scrupulous designing step and the comprehensive optimization of synthetic protocols are required to obtain the most effective sorbent. Alongside the experimental optimization, a theoretical analysis could be used as versatile tool to rationalize the process and to explain the behaviour of the material [14, 15].

The analysis of nitratively-altered macromolecules in biological samples has attracted attention due to their role in the pathogenesis of various diseases, such as asthma, diabetes or neurological disorders [16]. The elevated levels of reactive nitrogen species in cells could lead to the damage of cellular components, provoking pathological actions. In proteins, most of nitration reactions appear on a tyrosine aromatic ring, which is easily nitrated to 3-nitrotyrosine [17-22]. This compound is characterized by higher acidity than tyrosine, which affects the conformational stability of protein. As a result, an accelerated degradation of modified proteins is observed and higher levels of free 3-nitrotyrosine are detected, together with its main metabolite, 4-hydroxy-3-nitrophenylacetic acid (NHPA), which is excreted by urine. However, the NHPA is not exclusively formed from 3-nitrotyrosine. Pannala and co-workers [23] found that the levels of NHPA could double by the endogenous nitration of 4-hydroxyphenylacetic acid, present in saliva. The conversion of 4-hydroxyphenylacetic acid to NHPA occurs in the stomach, following the consumption of nitrate-rich products. Thus, it was stated that the urinary NHPA levels may be a good marker for the formation and metabolism of 3-nitrotyrosine, but dietary factors must be taken into account, assessing the nitroxidative stress products in vivo. The conversion of 4-hydroxyphenylacetic acid could also take place during in vitro analysis, affecting analytical results. Thus, to verify the hypothesis that the conditions of the analytical process could lead to an increased level of NHPA, the new MIP was designed. It was applied in the solid phase extraction (SPE) as a selective sorbent for the purpose of in vitro analysis of the conversion of 4-hydroxyphenylacetic acid to NHPA in human urine samples, under nitrite/nitrate-low $\mathrm{pH}$ conditions. The process was supported by theoretical analysis, the physicochemical characterization and SPE optimization.

\section{Experimental section}

\section{Materials and standards}

The templates and analytes: NHPA, 4-hydroxyphenylacetic acid (HPA), phenylacetic acid (PHE), 3-nitrobenzoic acid (NBA), 2-nitrophenol (NFE), (2S) 2-amino-3-(4-hydroxy-3-nitrophenyl)propanoic acid (3-nitro-L-tyrosine, 3NT), (2S) 2-amino-3-(4-hydroxy3-chlorophenyl)propanoic acid (3-chloro-L-tyrosine, 3CT), and 3,4-dimethoxyphenylacetic acid, the internal standard (IS) was from Sigma-Aldrich (Steinheim, Germany). The functional monomers: methacrylic 
acid (1) was from Sigma-Aldrich (Steinheim, Germany), allylamine (2), 4-vinylpyridine (3), 1-allyl-2thiourea (4) and 1-vinylimidazole (5) were from Fluka (Steinheim, Germany). The cross-linker, ethylene glycol dimethacrylate (EGDMA) was purchased from Sigma-Aldrich (Steinheim, Germany). The solvents: methanol, toluene, acetone, $t$-butyl-methyl ether and ammonium hydroxide were from POCh (Gliwice, Poland), the hexane and formic acid were from Merck (Darmstadt, Germany) and the ethyl acetate was delivered from Fluka (Steinheim, Germany). The polymerization reaction initiator, 1,1'azobiscyclohexanecarbonitrile, was from SigmaAldrich (Steinheim, Germany). The HPLC gradientgrade methanol and acetonitrile were purchased from Merck (Darmstadt, Germany). Ultra-pure water, delivered from a Hydrolab HLP 5 system (Straszyn, Poland), was used to prepare the water solutions.

Human urine samples were obtained from a healthy volunteer (a woman, of 20 years of age), who was enrolled in this study after signing a written informed consent. The subject, a non-smoker, did not consume alcohol or take any medication within two weeks of the start of the study. The urine sample was collected in the morning, after overnight fasting.

\section{Polymers}

Radical bulk polymerization was performed to obtain the polymers coded as MIP1-MIP5, imprinted by HPA, PHE, NBA and NFE-codes are extended in subscript. The templates acted as structural analogues of NHPA in a so-called pseudo-template approach [11]. The corresponding non-imprinted polymers, NIP1-NIP5, were prepared in the same way but without the template. The experimental quantities of the reagents, used for the preparation of polymers, are listed in Supplementary Information (Table S1).

Briefly, the chosen template, the appropriate functional monomer and the EGDMA were dissolved in a mixture of toluene and methanol $(3: 2 \mathrm{v} / \mathrm{v})$ in a thickwalled glass tube. The molar ratio of the template to the functional monomer and the cross-linker was 1:4:20. Finally, the polymerization initiator was added. The homogeneous solutions were purged with nitrogen for around five minutes (min.), then the glass tubes were sealed. Subsequently, polymerization was carried out in a nitrogen atmosphere for $24 \mathrm{~h}$ at $88{ }^{\circ} \mathrm{C}$. The bulk rigid polymers were ground and wet-sieved into particles below $45 \mu \mathrm{m}$ in diameter. Fine particles were separated by repeated decantation from acetone. The templates were removed from the polymers, using continuous extraction in a Soxhlet apparatus $(24-36 \mathrm{~h}, 80 \mathrm{~mL}$, methanol), followed by a washing sequence with $1 \%$ aq. ammonium hydroxide in methanol $(5 \mathrm{~mL} / 10 \mathrm{mg}$ of sorbent) and methanol (5 mL/10 mg of sorbent). The template removal was monitored by liquid chromatography, coupled with tandem mass spectrometry (LC-MS/MS). The particles were dried in a vacuum at room temperature.

\section{Instruments}

Instrumental analysis was performed using an Agilent 1260 Infinity System (Agilent Technologies, Santa Clara, CA, USA), equipped with a degasser, an autosampler and a binary pump, coupled to a QTRAP 4000 hybrid triple quadrupole/linear ion trap mass spectrometer (AB Sciex, Framingham, MA, United States). The turbo ion spray source was operated in negative mode. The curtain gas, ion source gas 1 and ion source gas 2, were set at $241 \mathrm{kPa}$, $414 \mathrm{kPa}, 276 \mathrm{kPa}$ and "high" instrument units $\left(4.6 \times 10^{-5}\right.$ Torr), respectively. The ion spray voltage and source temperature were $4,500 \mathrm{~V}$ and $600{ }^{\circ} \mathrm{C}$, respectively. The target compounds were analysed in multiple reaction monitoring (MRM) mode. The quantitative MRM transitions, declustering potential (DP) and collision energy (CE) are presented in the Supplementary Information (Table S2). Chromatographic separation was achieved with a Bionacom BionaCore C18 UFPLC Column $(100 \mathrm{~mm} \times 4.6 \mathrm{~mm}$, $2.7 \mu \mathrm{m}$ ) from Bionacom (Coventry, United Kingdom). The column was maintained at $40{ }^{\circ} \mathrm{C}$ at a flow rate of $0.5 \mathrm{~mL} \mathrm{~min}^{-1}$. The mobile phases consisted of water with $0.2 \%$ formic acid as eluent $\mathrm{A}$, and acetonitrile with $0.2 \%$ formic acid as eluent $\mathrm{B}$. The gradient $(\% \mathrm{~B})$ was as follows: $0 \mathrm{~min} .5 \%, 0.5 \mathrm{~min} .5 \%, 7 \mathrm{~min} .90 \%$ and $9 \mathrm{~min} .90 \%$. The re-equilibration of the column to the initial conditions lasted for $2 \mathrm{~min}$. The injection volume was $10 \mu \mathrm{L}$.

The method was characterized by acceptable precision (5.8\% within-run; $9.9 \%$ between-runs) and accuracy (110\% within-run; $114 \%$ between-runs). The limit of detection (LOD) and the limit of quantitation (LOQ), determined as the minimal concentration providing a peak height three and five times the 
baseline noise, was calculated in the urine as $85 \mathrm{ng}$ $\mathrm{L}^{-1}$ and $142 \mathrm{ng} \mathrm{L}^{-1}$, respectively.

The surface morphology analysis, using scanning electron microscopy (SEM) with a Merlin FE-SEM (Zeiss, Germany) and the X-ray electron dispersive spectroscopy (EDS) analysis, using an EDS X-ray detector (Brucker, Germany) were performed at the Faculty of Chemistry, University of Warsaw, Poland. The samples were Au/Pd sputter-coated, prior to the SEM analysis. The porosity data were determined using the adsorption isotherm of $\mathrm{N}_{2}$ at $77 \mathrm{~K}$ (BET) on an ASAP 2420 system (Micromeritics Inc., USA) at the Faculty of Chemistry, Maria Curie-Skłodowska University, Lublin, Poland. The infra-red (FT-IR) spectra were recorded on a Nicolet iS50 FT-IR (Thermo Fisher Scientific, Waltham, MA, United States) at the Biological and Chemical Research Centre, University of Warsaw, Poland. The thermogravimetry analyses (TGA) were performed at the Faculty of Chemistry, Warsaw University of Technology, Poland on a Q600 thermogravimetric analyser (TA Instruments, USA) in an argon atmosphere, with a heating rate of $5{ }^{\circ} \mathrm{C} \mathrm{min}$. Fluorescence detection was performed on the spectrofluorophotometer RF-6000 (Shimadzu, Duisburg, Germany), using a $10-\mathrm{mm}$ crystal cuvette. The 3D spectra were recorded using LabSolution software.

\section{Binding studies}

Stationary binding experiments were performed to evaluate the binding abilities of the MIPs and the NIPs in relation to the NHPA. These were carried out as previously described [24]. Regarding the NHPA, polypropylene tubes with a volume of $10 \mathrm{~mL}$ were filled with $10 \mathrm{mg}$ of $\mathrm{MIP} \mathbf{1}_{\mathbf{H P A}} / \mathrm{MIP}_{\mathbf{P H E}} / \mathrm{MIP}_{\mathbf{N B A}} /$ $\mathrm{MIP1}_{\mathrm{NFE}}-\mathrm{MIP}_{\mathrm{HPA}} / \mathrm{MIP}_{\mathrm{PHE}} / \mathrm{MIP}_{\mathrm{NBA}} / \mathrm{MIP}_{\mathrm{NFE}}$ or NIP1-NIP5 particles. A volume of $5 \mathrm{~mL}$ of $100 \mu \mathrm{g}$ $\mathrm{L}^{-1}$ methanol-water $(85: 15 \mathrm{v} / \mathrm{v})$ standard solution of NHPA was added to each tube. The tubes were sealed and oscillated, using a shaker at room temperature for $2 \mathrm{~h}$. Then, the tubes were centrifuged, and the aliquots of supernatant were used to analyse the unbound amounts of each compound by LC-MS/ MS, using the calibration lines. The amounts of NHPA bound to each polymer were calculated by subtracting the unbound amounts from the initial amounts.

For the isotherm analysis, the same procedure was applied to the MIP5 $5_{\text {HPA }}$ and NIP5 with the addition of different concentrations of NHPA in methanolwater $(85: 15 \mathrm{v} / \mathrm{v})$, ranging between 25 and $1000 \mu \mathrm{g}$ $\mathrm{L}^{-1}$.

The aforementioned selectivity studies were performed on the MIP5 $5_{\mathrm{HPA}}$ in the non-competitive experiments for the analytes: HPA, PHE, NBA, NFE, 3NT, 3CT. The polypropylene tubes, with a volume of $10 \mathrm{~mL}$, were filled with $10 \mathrm{mg}$ of $\mathrm{MIP}_{\mathrm{HPA}}$, and a volume of $5 \mathrm{~mL}$ of $7.5 \mu \mathrm{mol} \mathrm{L}{ }^{-1}$ methanol-water $(85: 15 \mathrm{v} / \mathrm{v})$ standard solution of each analyte, was added to each tube.

\section{Solid phase extraction}

For the optimization of the SPE process, an empty SPE 1-mL cartridge was filled with a mass of $10 \mathrm{mg}$ of MIP5 $5_{\text {HPA }}$ and secured by fibreglass frits. Subsequently, the following steps of the optimization of SPE were carried out as follows: conditioning with $1 \mathrm{~mL}$ of water, adjusted to $\mathrm{pH} 4$, loading of $1 \mathrm{~mL}$ of human urine (adjusted to $\mathrm{pH} 4$ ), spiked with NHPA

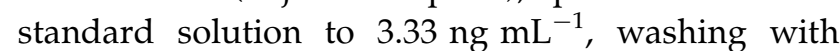
$1 \mathrm{~mL}$ of water, adjusted to $\mathrm{pH} 4$ prior to adding $1 \mathrm{~mL}$ of hexane or $15 \%$ methanol in water or $15 \%$ methanol in water, adjusted to $\mathrm{pH} 4$ or $15 \%$ methanol in water with $1 \%$ of ammonium hydroxide (before applying each solvent the sorbent was air-dried for $5 \mathrm{~min}$.) followed by eluting either by $t$-butyl-methyl ether, ethyl acetate or methanol. Following elution, the solvent was evaporated to dryness and was reconstituted in $100 \mu \mathrm{L}$ of methanol-water $(1: 1 \mathrm{v} / \mathrm{v})$ with IS (concentration of $100 \mathrm{ng} \mathrm{mL}^{-1}$ ), prior to analysis on the UPLC-MS/MS system. The recovery was calculated, based on an analysis of the unspiked sample of human urine, which was carried out in the same manner as described above, except for the addition of $70 \mu \mathrm{L}$ of IS and $33 \mu \mathrm{L}$ of NHPA standard solution (concentration of $100 \mathrm{ng} \mathrm{mL}^{-1}$ ) to eluate.

\section{Analysis of HPA conversion}

For the purpose of analysis of the HPA conversion to a volume of $900 \mu \mathrm{L}$ of human urine, adjusted to a physiological $\mathrm{pH}$ of the gastric environment $(\mathrm{pH} 2), \mathrm{a}$ volume of $100 \mu \mathrm{L}$ of sodium nitrite (concentration of

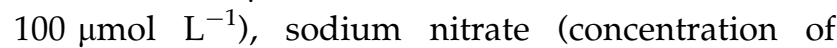
$10 \mathrm{mmol} \mathrm{L}{ }^{-1}$ ) and HPA (concentration of $100 \mathrm{mg}$ $\mathrm{L}^{-1}$ ) were added. The mixture was left for $30 \mathrm{~min}$. prior to SPE on the MIP5 $5_{\mathrm{HPA}}$, according to the optimized protocol. The eluates were collected, and the 
solvent was evaporated to dryness and was reconstituted in $100 \mu \mathrm{L}$ of methanol-water $(1: 1 \mathrm{v} / \mathrm{v})$ prior to analysis on the UPLC-MS/MS system. The human urine samples were analysed prior to testing, to assess the level of NHPA.

\section{Theoretical simulations}

All atom molecular dynamic (MD) simulations were performed using the BIOVIA Discovery Studio 2019 software package [25]. Before the molecular simulations of the prepolymerization, polymerization and adsorption processes, the optimization of the molecular structure and so-called ESP (electro-static potential) charges, a calculation of all compounds was performed using density functional theory (DFT) with a B3LYP/6-311 + G(d,p) hybrid functional, implemented in the Gaussian 09 program [26]. For all molecular systems, the construction and simulation processions were carried out and parametrized with a CHARMM force field [27]. Simulated systems were initially constructed using the Packmol software [28] to obtain random starting geometries. Firstly, the systems were energy-minimized (molecular mechanics (MM) method) with 100 steepest descent and 10,000 conjugate gradient steps to remove bad contacts. The processes were applied until the RMS (root-mean-square) gradient of the structure fell below $0.01 \mathrm{kcal} \mathrm{mol}^{-1} \AA^{-1}$. The MD protocols contained a heating step, performed for $100 \mathrm{ps,} \mathrm{with} \mathrm{time}$ steps of $1 \mathrm{fs}$. The system was heated from 0 to $300 \mathrm{~K}$. Prior to the production stage, isothermal equilibration was performed for 100 ps, at a temperature of $300 \mathrm{~K}$. The Leapfrog Verlet integration and SHAKE [29] algorithms were used during the simulation process. The production run was conducted for $5 \mathrm{~ns}$ in the NVT ensemble (constant-volume/constanttemperature dynamics) at $300 \mathrm{~K}$, and the coordinates were recorded every $10 \mathrm{ps}$. Trajectory file data, generated from the NVT MD simulation, have been used in all the calculations and analyses presented in this research.

In the first stage of polymers creation, the models of prepolymerization complexes (coded as PC) were constructed between the template and the monomers. To obtain the starting structures, the boxes with the chosen template molecule (HPA, PHE, NBA or NFE), surrounded by four molecules of appropriate monomer molecules (1) - (5) were created. Next, 20 molecules of the cross-linker and an appropriate number of solvent molecules (Table S1) were added, and complete starting structures were created using Packmol. Next, MM and MD procedures were applied to obtain models of prepolymerization systems with intermolecular interactions, formed between the template and monomer or cross-linking agents. The number of reagents and solvent molecules were chosen to mimic their molar ratio, used during the synthetic process.

In the next stage of the modelling procedure, the creation of polymer chains with the binding sites was performed (coded as MIP). The optimized structures of the prepolymerization complexes with minimum potential energy values, were chosen for further investigation. Single bonds between vinyl groups of monomer and cross-linker molecules in the prepolymerization systems were created. The $C$ atoms of the monomer or cross-linker vinyl groups which were closest to each other were connected, but also took into account the fact that all molecules of the monomer and cross-linker in the studied system, should create one cross-linked polymeric chain. Then, the $\mathrm{H}$ atoms were added to the chain structure. The operation described, mimicked the polymerization reaction in the synthetic process. So called ESP charges were calculated for the creation of the polymeric chain structures. Next, the MM and MD procedures were repeated for the constructed systems, which consisted of the polymeric chain, the template and the solvent, to form a specific binding site in the polymeric matrix.

In the last step of computational calculations, the adsorption process was simulated. The optimized structures of the polymeric chains with the minimum potential energy values, were selected for further investigation. The template was removed from the models of cavities and the empty spaces were proposed as the computer models of binding sites. Then, the molecule of the NHPA was inserted into the models of the MIPs' cavities (replacing the template molecule). The solvent, consisting of 418 molecules of methanol and 167 molecules of water, was added to form starting structures using Packmol, then the MD simulations were carried out (mimicking the experimental conditions of adsorption). During selectivity studies, the chosen analytes, namely HPA, PHE, NBA, NFE, 3NT and 3CT were inserted into the MIP5 $_{\text {HPA }}$ cavity model, using the same solvent as was applied to the NHPA adsorption studies. Then 
Table 1 Recovery of NHPA after SPE of human urine spiked to $3.33 \mathrm{ng} \mathrm{mL}^{-1}$ in the NHPA standard solution on MIP5 ${ }_{\text {HPA }}(\mathrm{n}=3$ )

\begin{tabular}{|c|c|c|c|}
\hline \multirow{2}{*}{$\begin{array}{l}\text { Solvent } \\
\text { Eluting } \\
\text { Washing }\end{array}$} & \multicolumn{3}{|l|}{ Recovery (\%) } \\
\hline & $t$-Butyl-methyl ether & Ethyl acetate & Methanol \\
\hline Water adjusted to $\mathrm{pH} 4$ & $31 \pm 3$ & $60 \pm 7$ & $83 \pm 21$ \\
\hline Hexane & $39 \pm 3$ & $78 \pm 14$ & $84 \pm 1$ \\
\hline $15 \%$ Methanol in water & $29 \pm 2$ & $58 \pm 14$ & $62 \pm 7$ \\
\hline $15 \%$ Methanol in water adjusted to $\mathrm{pH} 4$ & $38 \pm 4$ & $68 \pm 6$ & $75 \pm 21$ \\
\hline $15 \%$ Methanol in water with $1 \%$ of ammonium hydroxide & $3 \pm 1$ & $3 \pm 1$ & $4 \pm 1$ \\
\hline
\end{tabular}

starting structures were obtained and finally, the MD calculations were performed.

\section{Calculation of experimental and theoretical binding parameters}

The binding capacities $\left(B, \mu \mathrm{g} \mathrm{g}^{-1}\right)$ of the MIPs and NIPs are calculated according to Eq. 1:

$B=\frac{\left(C_{i}-C_{f}\right) V}{m}$

The distribution coefficients $\left(K_{\mathrm{D}}, \mathrm{L} \mathrm{g}^{-1}\right)$ for the MIPs and NIPs are calculated according to Eq. 2:

$K_{D}=\frac{\left(C_{i}-C_{f}\right) V}{C_{f} m}$

where $V$ represents the volume of the solution (L), $C_{\mathrm{i}}$ represents the initial solution concentration $\left(\mu \mathrm{g} \mathrm{L}^{-1}\right)$, $C_{\mathrm{f}}$ represents the solution concentration after adsorption $\left(\mu \mathrm{g} \mathrm{L}^{-1}\right)$ and $m$ is the mass of the particles (g). The affinity factors (AF) are obtained according to Eq. 3:

$A F=\frac{K_{D(\mathrm{MIP})}}{K_{D(\mathrm{NIP})}}$

The adsorption isotherms were characterized using the Langmuir model, transformed into the Scatchard equation, as shown in Eq. 4 :

$\frac{B}{F}=\frac{\left(B_{\max }-B\right)}{K_{d}}$

where $B_{\max }$ is the total number of binding sites, $K_{\mathrm{d}}$ is the dissociation constant, $B$ is the bound amount of the analyte and $F$ is the unbound amount of the analyte. The system, which fits well with a Langmuir model, gives a straight line on the Scatchard plot, with a slope equal to $-\left(1 / K_{\mathrm{d}}\right)$ and a $y$-intercept, equal to $B_{\max } / K_{\mathrm{d}}$. It also fits with the Freundlich model, represented by Eq. 5:

$B=a F^{m}$

where $B$ is the bound amount of the analyte, $F$ is the unbound amount of the analyte, $a$ is a measure of the capacity $\left(B_{\max }\right)$ and $m$ is a heterogeneity index.

\section{Results and discussion}

\section{Design of sorbent}

Different functional monomers and templates were analysed in the material designing step. A set of 20 MIPs were synthesized from five different monomers, viz. (1)-(5) involving four different templates, viz. HPA, PHE, NBA and NFE. These templates acted as structural analogues of the target analyte, NHPA, in the so-called pseudo-template strategy [11]. The NIPs were also obtained.

\section{Effect of functional monomer on binding capacity in relation to the NHPA}

Firstly, the determination of $B$ of $M I P 1_{\mathbf{H P A}} /$ $\mathrm{MIP1}_{\mathrm{PHE}} / \mathrm{MIP1}_{\mathrm{NBA}} / \mathrm{MIP1}_{\mathrm{NFE}}-\mathrm{MIP}_{\mathrm{HPA}} / \mathrm{MIP}_{\mathrm{PHE}} /$ MIP5 $5_{\text {NBA }} / \mathrm{MIP}_{\mathrm{NFE}}$ and NIP1 - NIP5 in relation to the NHPA was carried out to evaluate the effect of the composition of the polymer network on the adsorption behaviour. The stationary experiments were carried out and the $B\left(\mu \mathrm{g} \mathrm{g}^{-1}\right)$ values of the MIPs and the NIPs were calculated, according to Eq. 1. The calculation of $K_{\mathrm{D}}\left(\mathrm{L} \mathrm{g}^{-1}\right)$, according to Eq. 2, followed by the calculation of AF according to Eq. 3, was also carried out (Table S3). Figure 1 presents the $B$ of $\mathrm{MIP}_{\mathrm{HPA}} / \mathrm{MIP}_{\mathrm{PHE}} / \mathrm{MIP1}_{\mathrm{NBA}} /$ 


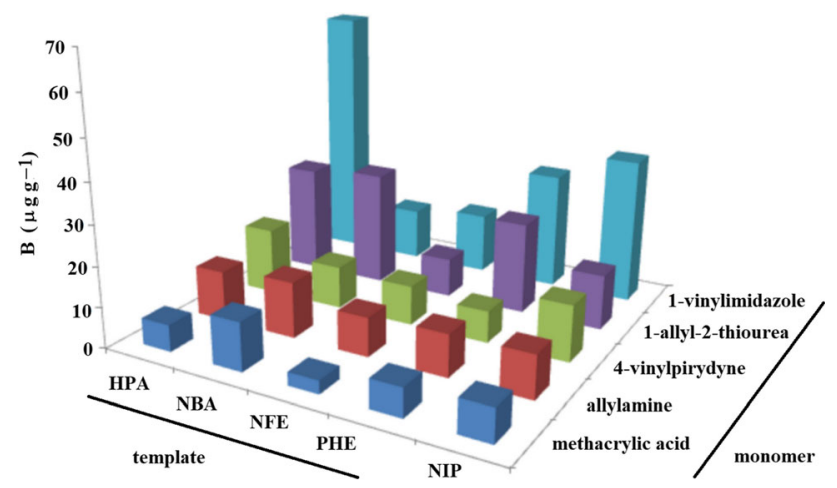

Figure 1 Binding capacities $(B)$ of $\mathrm{MIP} 1_{\mathrm{HPA}} / \mathrm{MIP} 1_{\mathrm{PHE}} / \mathrm{MIP} 1_{\mathrm{NBA}} /$ $\mathrm{MIP} 1_{\mathrm{NFE}}-\mathrm{MIP}_{\mathrm{HPA}} / \mathrm{MIP} 5_{\mathrm{PHE}} / \mathrm{MIP}_{\mathrm{NBA}} / \mathrm{MIP}_{\mathrm{NFE}}$ and NIP1NIP5 for the NHPA $\left(\mathrm{c}=100 \mu \mathrm{g} \mathrm{L}^{-1}, n=3\right.$, RSD $\left.<4.5 \%\right)$.

$\mathrm{MIP}_{\mathrm{NFE}}-\mathrm{MIP}_{\mathrm{HPA}} / \mathrm{MIP}_{\mathrm{PHE}} / \mathrm{MIP}_{\mathrm{NBA}} / \mathrm{MIP} 5_{\mathrm{NFE}}$ and NIP1-NIP5 in relation to the NHPA.

As can be seen (Fig. 1), the lowest $B$ with regard to the NHPA were noted in the case of MIPs prepared from (1) (from 3.30 to $12.2 \mu \mathrm{g} \mathrm{g}^{-1}$ for MIP1 $\mathbf{N F E}_{\mathrm{NFE}}$ and MIP1 $1_{\text {NBA }}$, respectively). Only slightly higher values of $B$ were observed for polymers prepared from (2) (from 9.81 to $14.0 \mu \mathrm{g} \mathrm{g}^{-1}$ for MIP2 $\mathbf{N F E}_{\mathrm{NFE}}$ and MIP2 $\mathbf{N B A}_{\mathrm{NB}}$ respectively) and (3) (from 7.96 to $16.6 \mu \mathrm{g} \mathrm{g}^{-1}$ for $\mathrm{MIP}_{\mathrm{PHE}}$ and $\mathrm{MIP3}{ }_{\mathrm{HPA}}$, respectively). Significantly higher $B$ were noted in the case of the MIPs synthesized from (4) (from 9.83 to $26.90 \mu \mathrm{g} \mathrm{g}^{-1}$ for MIP4 $4_{\mathrm{NFE}}$ and MIP1 $_{\mathbf{H P A}}$, respectively). The highest values of $B$ characterized the MIPs build up from (5), acting as the functional monomer (with the highest $B$ among all tested polymers for MIP5 $5_{\mathrm{HPA}}$, equal to $64 \mu \mathrm{g} \mathrm{g}^{-1}$ ). A similar trend was observed for the NIPs. Those results showed that the adsorption process of the NHPA strongly depended on the composition of the polymer and (5) was the most effective monomer among all tested compounds, in providing MIPs in relation to the NHPA. These results also concurred with the observations of Baggiani and co-workers [30] who postulated that if the NIP indicated binding properties towards a target analyte, the similarly composed MIP would reveal a higher $B$ and a significant imprinting effect.

Thus, in the next step, the impact of different templates and the structural analogues of the target analyte on the specificity of resulting MIPs was investigated.
Effect of template on specificity

As shown (Table S3), the highest specificity (expressed as $\mathrm{AF}$ ) was noted for $\mathrm{MIP} 5_{\mathrm{HPA}}$, prepared from (5) in the presence of HPA with $\mathrm{AF}=3.03$. The polymers prepared from (4) also revealed satisfactory specificity; MIP4 $4_{\mathrm{NBA}}$ was prepared in the presence of NBA as the template was characterized by sufficient specificity, $\mathrm{AF}=2.51$, together with $\mathrm{MIP}_{\mathrm{PHE}}$, prepared in the presence of PHE, $\mathrm{AF}=1.84$. The results could be explained by the structure of the template and its interactions with respective monomers. It could be postulated that the interactions between the imidazole nitrogen atoms and the methylene carboxylic group from the HPA (which is characterized by higher acidity when compared to NBA) efficiently stabilized the prepolymerization complexes. The remaining tested systems failed to provide the satisfactory specificity of the resulting MIPs.

Thus, to reveal the molecular bases of the interactions between analysed templates and monomers, as well as to understand the mechanism of adsorption, a theoretical analysis was carried out.

\section{Theoretical proof of specificity}

In order to provide an insight into the mechanism that governs the adsorption of NHPA on selected polymeric matrices, the following analyses were carried out: (a) the analysis of interactions between the respective templates, monomers and cross-linker in the prepolymerization complexes of $\mathrm{PC} 5_{\mathrm{HPA}}$, $\mathrm{PC}_{\mathrm{NBA}}, \mathrm{PC} \mathbf{4}_{\mathrm{PHE}}, \mathrm{PC} \mathbf{1}_{\mathrm{NBA}}$ and $\mathrm{PC} \mathbf{1}_{\mathrm{NFE}}$ (it imitates the formation process of the complex), (b) the analysis of interaction of the template (HPA, NBA, PHE or NFE) in the cavities of $\mathrm{MIP}_{\mathrm{HPA}}, \mathrm{MIP}_{\mathrm{NBA}}, \mathrm{MIP}_{\mathrm{PHE}}$, $M I P 1_{\mathrm{NBA}}$ and MIP1 $1_{\mathrm{NFE}}$ (it imitates the interaction of the template in polymerized systems only), (c) the analysis of interactions of NHPA in the cavities of $\mathrm{MIP5}_{\mathrm{HPA}}, \mathrm{MIP}_{\mathrm{NBA}}, \mathrm{MIP} 4_{\mathrm{PHE}}, \mathrm{MIP} \mathbf{1}_{\mathrm{NBA}}$ and MIP1 $1_{\mathrm{NFE}}$ (it imitates the process of adsorption of the target analyte inside the cavity, after template removal). Those five systems were selected taking into account the following: the highest $B$ and specificity $\left(\mathrm{PC}_{\mathrm{HPA}} /\right.$ MIP5 $\left.5_{\mathrm{HPA}}\right)$, the second and third highest specificity $\left(\mathrm{PC}_{\mathrm{NBA}} / \mathrm{MIP}_{\mathrm{NBA}}\right.$ and $\left.\mathrm{PC} 4_{\mathrm{PHE}} / \mathrm{MIP}_{\mathrm{PHE}}\right)$, the fourth highest specificity but low $B\left(\mathrm{PC}_{\mathrm{NBA}} /\right.$ MIP1 $\left.1_{\text {NBA }}\right)$, as well as the lowest $B$ and lack of specificity $\left(\mathrm{PC} \mathbf{1}_{\mathrm{NFE}} / \mathrm{MIP} \mathbf{1}_{\mathrm{NFE}}\right)$. The impact of the solvent on the interactions is also discussed. 


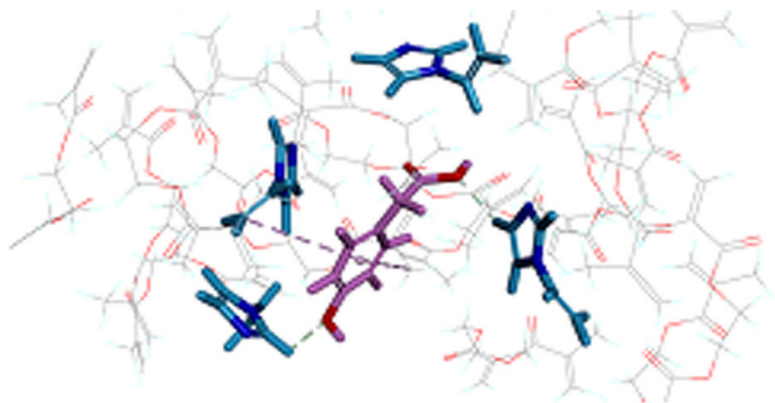

(a)

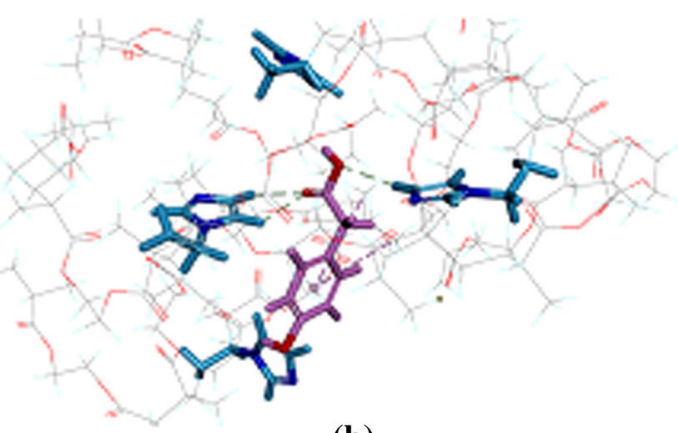

(b)

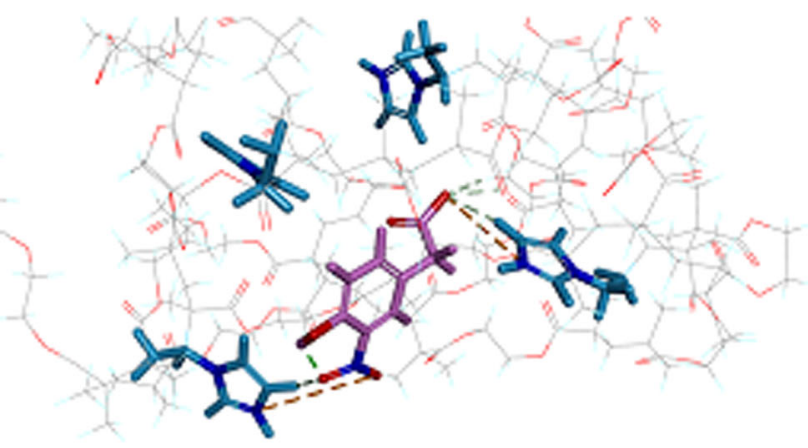

(c)

Figure 2 Views of theoretically generated complexes of $\mathrm{PC} 5_{\mathrm{HPA}}$ (a), template of HPA (b) and analyte NHPA in the cavity of $\mathrm{MIP5}_{\mathrm{HPA}}$ (c).

In the PC5 $5_{\text {HPA }}$ model (Fig. 2a), two types of interactions between the template (HPA) and the monomer (5) or cross-linker were observed, viz. hydrogen bonds and hydrophobic interactions. Two non-classical hydrogen bonds were formed between the $\mathrm{O}$ atom from the $-\mathrm{COOH}$ or $-\mathrm{OH}$ groups of the HPA, and the $\mathrm{H}$ atoms from the imidazole rings of the two monomer molecules, whereas two hydrophobic interactions $(\pi$-alkyl) were observed between the aromatic ring of the HPA and the $-\mathrm{CH}_{2}-$ group of (5) or the $-\mathrm{CH}_{3}$ group of the EGDMA. Additionally, the interactions of the HPA with the solvent molecules were present (a $\pi-\pi$ T-shaped interaction with a toluene ring, as well as a hydrogen bond, and a $\pi$ lone pair interaction with methanol). After mimicking the polymerization process, the model of the

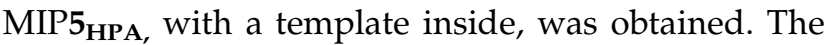
types of interactions between the HPA and the chain model of the MIP5 $5_{\text {HPA }}$ (just after imitated polymerization) were the same as those in the PC $5_{\mathrm{HPA}}$ (Fig. 2b) but the number of interactions varied. Here, three non-classical hydrogen bonds were formed between the $\mathrm{O}$ atoms from the $-\mathrm{COOH}$ group of the HPA and the $\mathrm{H}$ atoms from the imidazole rings of two monomer residues in the polymeric chain. Two hydrophobic interactions ( $\pi$-alkyl) were also noted between the aromatic ring of the HPA and the $-\mathrm{CH}_{3}$ groups from one EGDMA residue. Additionally, the HPA interacted with only one molecule of the solvent, viz. toluene, creating three hydrophobic interactions ( $\pi$ - $\pi$ T-shaped, $\pi$-sigma and $\pi$-alkyl type). In the final step, the template molecule of the HPA was removed from the system (mimicking template removal step) and the empty cavity of the MIP5 $5_{\text {HPA }}$ was obtained. Next, the adsorption process of the NHPA (target analyte) on the MIP5 $5_{\text {HPA }}$ matrix was simulated (Fig. 2c). Following the MD procedure, three non-classical hydrogen bonds were formed between the NHPA $\left(\mathrm{O}\right.$ atoms of $-\mathrm{COOH}$ or $-\mathrm{NO}_{2}$ groups) and the $\mathrm{H}$ atoms from the imidazole rings of the two monomer residues or the $-\mathrm{CH}_{3}$ group from the EGDMA residue. However, it should be underlined that the experimental process of adsorption was carried out in aqueous solution. Thus, the possibility of the ionization of the imidazole groups and the carboxyl group of the NHPA, was taken into account. Hence, the creation of two electrostatic interactions between the $\mathrm{N}$ atoms from the imidazole rings and the $\mathrm{O}$ atoms from the $-\mathrm{COO}^{-}$or $-\mathrm{NO}_{2}$ groups of NHPA, as well as the formation of an additional, non-classical hydrogen bond between the $\mathrm{O}$ atom from the $-\mathrm{COO}^{-}$and the $\mathrm{H}$ atom from the $\mathrm{CH}_{3}$ group of the EGDMA residue were observed.

In the $\mathrm{PC}_{\mathrm{NBA}}$ system, five interactions between the NBA and the monomers or cross-linker were formed: one hydrogen bond between the $\mathrm{H}$ atom from the $-\mathrm{COOH}$ group of the NBA and the $\mathrm{O}$ atom of the EGDMA, two hydrophobic ( $\pi$-alkyl) and two $\pi$ sulphur-type interactions between the aromatic ring of the NBA and the vinyl groups or $\mathrm{S}$ atoms of three monomers. Two interactions $(\pi-\pi$ stacked and $\pi$ alkyl) between the aromatic ring of the NBA and only one solvent molecule (toluene) were also present. 
After the simulation of the polymerization process, only one $\pi$-sulphur-type interaction between the NBA and the $S$ atom of the monomer residue from the polymeric chain was observed, whereas three hydrophobic interactions (two $\pi$-alkyl and one $\pi-\pi$ stacked type) with two toluene molecules were formed. Finally, the adsorption of the NHPA on the MIP4 $4_{\text {NBA }}$ cavity after NBA removal, revealed seven interactions between the analyte and the polymer chain. Here, five hydrogen bonds were formed between either the $\mathrm{O}$ or $\mathrm{H}$ atoms from the $-\mathrm{COOH}$ group or the $\mathrm{O}$ atom from the $-\mathrm{NO}_{2}$ group of the NHPA and the $\mathrm{H}$ atoms from the $-\mathrm{NH}-$ or $-\mathrm{NH}_{2}$ groups, or the $\mathrm{S}$ atoms of the three monomer residues, or the $\mathrm{O}$ atom from the EGDMA residue. Additionally, two non-classical hydrogen bonds between the $\mathrm{O}$ atoms from the $-\mathrm{COOH}$ or $-\mathrm{NO}_{2}$ group of the NHPA and the $-\mathrm{CH}_{2}$ - group of the EGDMA or monomer residue, and one $\pi$-sulphurtype interaction between the analyte and the monomer residue were observed.

In the analysis of the PC4 $4_{\mathrm{PHE}}$ system, three interactions between the template and the monomers or the EGDMA were observed, viz. two hydrophobic ( $\pi$ alkyl) interactions and one $\pi$-sulphur-type interaction, created between the aromatic ring of the PHE and the $-\mathrm{CH}_{3}$ groups of the EGDMA, or the $\mathrm{S}$ atom of (4). Additionally, two non-classical hydrogen bonds between the $\mathrm{O}$ atoms from the $-\mathrm{COOH}$ group of the PHE and the solvent molecule of methanol were formed. During the analysis of the polymeric system, with the chain of MIP4 $\mathrm{PHE}$ and the template, more interactions were found between the template and the polymer immediately after the simulation of the polymerization process, than in the prepolymerization complex. One classical and one non-classical hydrogen bond were created between the $\mathrm{O}$ atoms from the $-\mathrm{COOH}$ group of the $\mathrm{PHE}$ and the $\mathrm{H}$ atoms from the $-\mathrm{NH}_{2}$ or $-\mathrm{CH}_{2}$ - group of the two monomer residues; four hydrophobic interactions $(\pi$-alkyl) were observed between the PHE and the $-\mathrm{CH}_{2}-$ or $\mathrm{CH}_{3}$ groups of the two EGDMA residues, and one $\pi$ sulphur-type interaction was also observed between the PHE and the (4) residue. One classical and one non-classical hydrogen bond were formed between the template and the solvent (methanol) molecules. After simulation of NHPA adsorption in the empty cavity of the MIP4 $4_{\mathrm{PHE}}$, one hydrogen bond between the $-\mathrm{NO}_{2}$ group of analyte and the $\mathrm{H}$ atom from the $\mathrm{NH}_{2}$ group of the monomer residue was found, together with five $\pi$-alkyl-type interactions of NHPA with the $-\mathrm{CH}_{2}-$ or $-\mathrm{CH}_{3}$ groups of the four EGDMA residues, as well as one $\pi$-sulphur-type interaction. Additionally, eight non-classical hydrogen bonds between the $\mathrm{O}$ atoms from the $-\mathrm{COOH}$ or $-\mathrm{NO}_{2}$ and the methanol molecules were also observed.

The template molecule of the NBA in the PC1 $\mathbf{1}_{\mathrm{NBA}}$ prepolymerization system created only two interactions with the EGDMA molecules, however, no interactions with the monomer were observed. One classical hydrogen bond was formed by the $\mathrm{H}$ atom from the - $\mathrm{COOH}$ group and one non-classical hydrogen bond was formed by the $\mathrm{O}$ atom from the $\mathrm{NO}_{2}$ group of the NBA. The template interacted with the solvent, forming one non-classical hydrogen bond with methanol, one $\pi-\pi$ stacked, two $\pi$-alkyl and one $\pi$-cation-type interaction with two toluene molecules. Following polymerization, the NBA in the chain of the MIP1 $1_{\text {NBA }}$ still had only one classical hydrogen bond with the EGDMA residue and three $\pi$-alkyl interactions with the $-\mathrm{CH}_{2}-$ or $-\mathrm{CH}_{3}$ groups of the EGDMA or (1) residues. Interactions of the NBA with the solvent were also present, viz. one classical hydrogen bond between the $\mathrm{O}$ atom from the $-\mathrm{NO}_{2}$ group of the NBA and methanol, two non-classical hydrogen bonds between the $\mathrm{O}$ atoms from the $\mathrm{COOH}$ or $-\mathrm{NO}_{2}$ groups and methanol, and $\pi-\pi$ stacked and $\pi$-alkyl interactions with the toluene molecule. After the simulation of the adsorption process of the NHPA on MIP1 $\mathbf{1}_{\mathrm{NBA}}$, two hydrogen bonds between the $\mathrm{H}$ atom from the $-\mathrm{COOH}$ group of analyte and the EGDMA residue, and between the $\mathrm{O}$ atom from the $-\mathrm{NO}_{2}$ group of analytes and the monomer residue were observed. Four hydrophobic ( $\pi$-alkyl) interactions of the NHPA with the $-\mathrm{CH}_{3}$ groups of the EGDMA or monomer residues were also present, as well as one non-classical hydrogen bond, formed between the $\mathrm{O}$ atom from the $-\mathrm{COOH}$ group of the NHPA and the $-\mathrm{CH}_{2}-$ group of the EGDMA residue. In this system, the analyte interacted with the solvent, creating three classical hydrogen bonds between the $\mathrm{O}$ atoms from the $\mathrm{COOH}$ or the $-\mathrm{NO}_{2}$ groups and the methanol or water molecules, and four non-classical hydrogen bonds with methanol.

In the $\mathrm{PC} \mathbf{1}_{\mathrm{NFE}}$ prepolymerization model, one nonclassical hydrogen bond between the $\mathrm{O}$ atom from the $-\mathrm{OH}$ group of the PHE and the $-\mathrm{CH}_{2}-$ group of the EGDMA molecule, and two hydrophobic ( $\pi$ alkyl) interactions of the PHE with the $-\mathrm{CH}_{3}$ groups 
of the two EGDMA molecules, were observed. No interactions of the template with the monomers were found. NFE also interacted with the toluene molecule, forming $\pi$ - $\pi$ T-shaped, $\pi$-alkyl and $\pi$-sigma interactions. After polymerization, the NFE interacted with the polymer chain of $\mathrm{MIP} \mathbf{1}_{\mathrm{NFE}}$, creating two non-classical hydrogen bonds between the $\mathrm{O}$ atoms of the $-\mathrm{OH},-\mathrm{NO}_{2}$ and $-\mathrm{CH}_{2}-$ groups of one EGDMA residue, and one $\pi$-alkyl interaction with the $-\mathrm{CH}_{2}-$ group of the monomer residue. The PHE formed three interactions: $\pi$ - $\pi$ stacked, $\pi$-anion and $\pi$ cation with the two solvent molecules of toluene. After the simulation of the NHPA adsorption process in the MIP1 $1_{\mathrm{NFE}}$, three types of interactions between the analyte and polymer chain were observed. Two hydrogen bonds were formed between the $\mathrm{O}$ or $\mathrm{H}$ atoms from the $-\mathrm{COOH}$ group of the NHPA and the $\mathrm{O}$ or $\mathrm{H}$ atoms from the $-\mathrm{COOH}$ groups of the two monomer residues. Two non-classical hydrogen bonds were created between the $\mathrm{O}$ atom from the $\mathrm{OH}$ group of the analyte and the $-\mathrm{CH}_{2}$ groups of one EGDMA residue. Three $\pi$-alkyl interactions were formed between the NHPA and the $-\mathrm{CH}_{2}-$ or $-\mathrm{CH}_{3}$ groups of monomer or EGDMA residues. The NHPA created numerous interactions with the solvent. Six classical hydrogen bonds were observed between the $\mathrm{O}$ atoms from the $-\mathrm{COOH}$ or the $-\mathrm{NO}_{2}$ groups of the NHPA and methanol, or water molecules; two nonclassical hydrogen bonds and two $\pi$-lone pair type interactions between the analyte and the methanol molecules were also present in the analysed system.

In summary, it should be emphasized that numerous interactions between the template and the monomer, supported by an additional interaction with the cross-linker and solvent, stabilized the prepolymerization complex. It could also be noted that the analysis of interactions between the template and the polymer chain revealed slight variations, when compared to interactions in the prepolymerization complex. Finally, considering the interactions of the NHPA analyte in tested cavities, it could be concluded that the $B$ decreased with the increasing number of interactions with solvent molecules.

On the basis of the experimental and theoretical results, the polymer MIP5 $_{\text {HPA }}$ was selected as the most appropriate candidate for further analysis.

\section{Characterization of sorbent}

\section{Morphology and structure}

In order to identify the surface morphologies of MIP5 $_{\text {HPA }} /$ NIP5, the analysis of micrographs from SEM was carried out. The micrographs are presented in Fig. 3.

The polymers were obtained by the thermal radical bulk polymerization. As can be seen, the micrographs of MIP5 $_{\text {HPA }}$ (Fig. 3a) and NIP5 (Fig. 3c) revealed a typical format of particles for bulk, crushed and sieved materials, with a high degree of irregularity, as well as heterogeneous morphology. A higher magnification of micrographs revealed negligible variations on the surface of MIP5 ${ }_{\mathrm{HPA}}$ and NIP5 (Fig. 3b, d). MIP5 ${ }_{\text {HPA }}$ was characterized by a more densely formed surface, with a lesser amount of detectable macropores.

To provide an insight into the pore structure, the nitrogen sorption analysis was used, and the data obtained, were inserted into the correct mathematical models. Firstly, the nitrogen adsorption isotherms (Brunauer-Emmett-Teller isotherm) were analysed for both MIP5 ${ }_{\text {HPA }}$ and NIP5 (Fig. 4) [31].

As shown, the tested materials indicated physisorption type IV isotherms that characterized mesoporous sorbents with unrestricted, mono-multilayered adsorption [32]. The hysteresis loops were also revealed with type $\mathrm{H} 3$ loops for $\mathrm{MIP}_{\mathrm{HPA}}$ and NIP5. This could indicate the slit-shaped structure of the pores of both materials obtained (Fig. 4a). Low pressure hystereses were observed for all materials, and the lack of closing points could be explained by the experimental conditions, rather than the porosity of the sorbents [33].

Next, the total specific surface area (BrunauerEmmett-Teller isotherm) was evaluated, together with the cumulative surface area of the pores (Barrett-Joyner-Halenda model), and the volume and area of the micropores (Harkins-Jura equation) $[34,35]$. A slightly larger, specific surface area was noted in the case of MIP5 $5_{\text {HPA }}\left(368.6 \mathrm{~m}^{2} \mathrm{~g}^{-1}\right)$ when compared with NIP5 $\left(365.2 \mathrm{~m}^{2} \mathrm{~g}^{-1}\right)$. This correlated well with the surface morphology analysis, revealing negligible variations (Fig. 3). The micropore area and the external surface area of MIP5 $_{\mathrm{HPA}}$ were also greater than those of NIP5 $\left(94.9,273.6 \mathrm{~m}^{2} \mathrm{~g}^{-1}\right.$ for MIP5 $_{\text {HPA }}$ and $87.5,267.7 \mathrm{~m}^{2} \mathrm{~g}^{-1}$ for NIP5, respectively). A higher micropore volume was noted for 
(a)

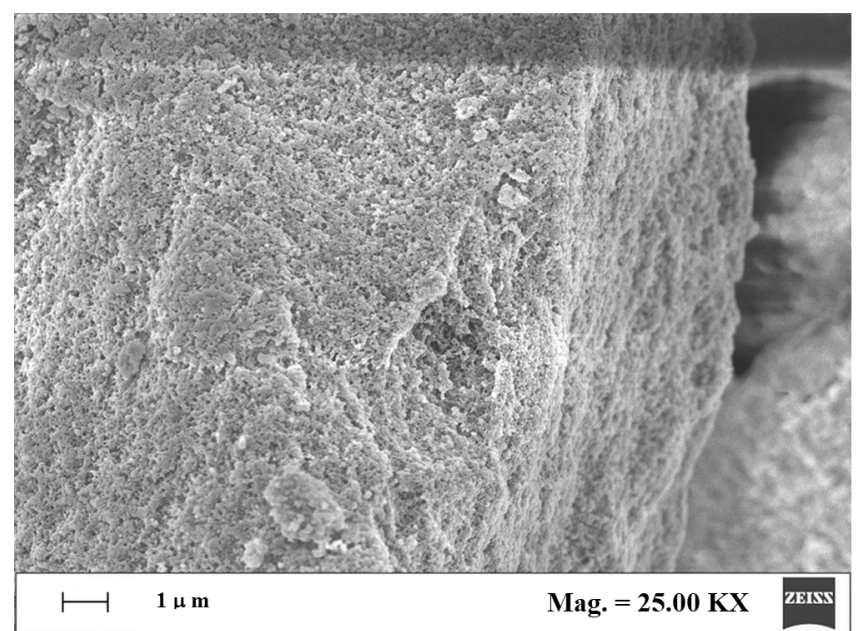

(c)

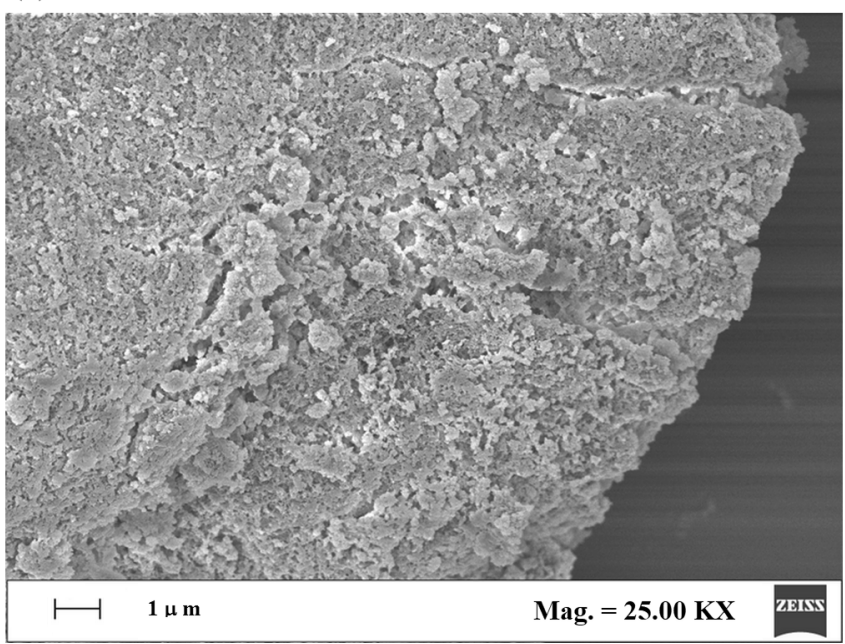

(b)

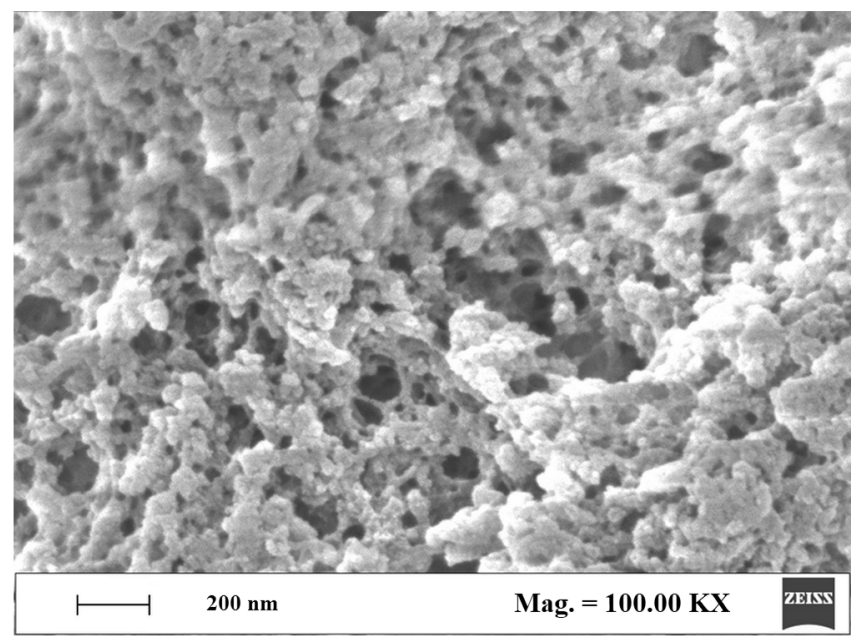

(d)

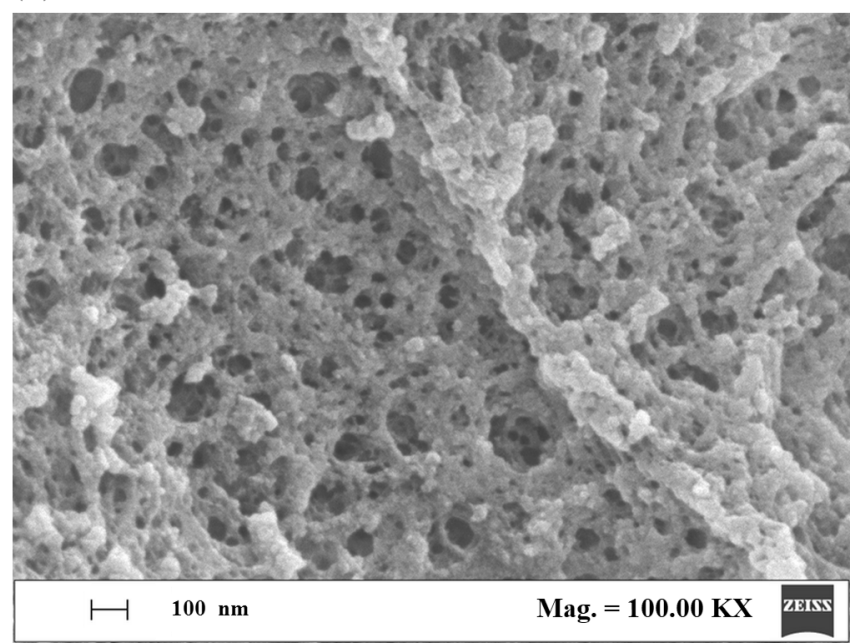

Figure 3 SEM micrographs of MIP5 $5_{\mathrm{HPA}}(\mathbf{a}, \mathbf{b})$ and $\operatorname{NIP5}(\mathbf{c}, \mathbf{d})$.

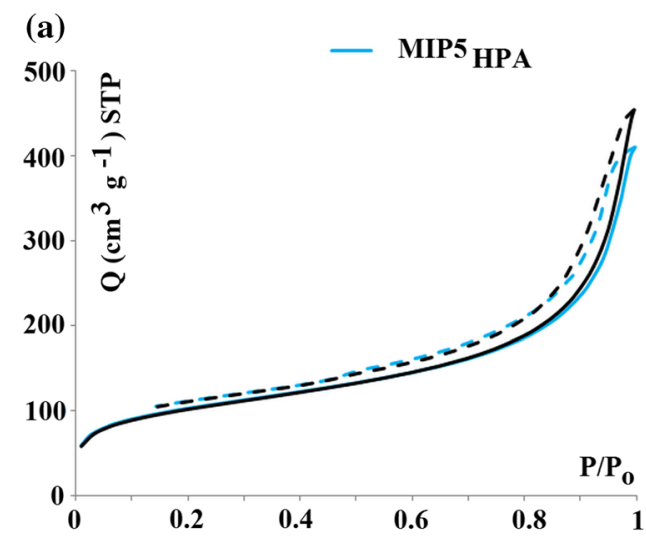

Figure 4 Nitrogen adsorption isotherms (left) for MIP5 $5_{\text {HPA }}$ (light blue) and NIP5 (black) - adsorption lines are continuous; desorption lines are dashed $\left(\mathrm{P}_{\mathrm{o}}\right.$ is the nitrogen saturation pressure). Pore size distributions (right), calculated using the (b)

- NIP5

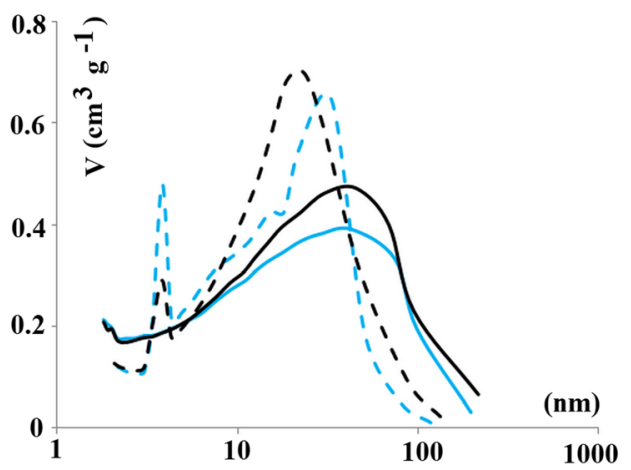

Barrett-Joyner-Halenda model for both the adsorption and the desorption branches of isotherms for MIP5 $5_{\mathrm{HPA}}$ (light blue) and NIP5 (black) - adsorption lines are continuous, desorption lines are dashed. 
MIP5 $_{\text {HPA }}$ than for NIP5 $\left(0.039\right.$ to $0.035 \mathrm{~cm}^{3} \mathrm{~g}^{-1}$ respectively) however, a higher total volume of pores was noted for NIP5, than for MIP5 ${ }_{\text {HPA }}$ (0.703 to 0.635 $\mathrm{cm}^{3} \mathrm{~g}^{-1}$ respectively). To provide a greater insight into the characterization of pore structures, the pore size distributions was analysed. Figure $4 \mathrm{~b}$ presents pore size distributions, calculated using the BarrettJoyner-Halenda model on both the adsorption and the desorption branches of the isotherms for both MIP5 $_{\text {HPA }}$ and NIP5.

The pore volumes, set against pore diameter plots and calculated from the desorption isotherms, revealed peaks at 33 and $23 \mathrm{~nm}$ for MIP5 $_{\mathrm{HPA}}$ and NIP5, respectively. Thus, both materials were characterized by the mesoporous structure of pores but a higher micropore area and external surface area of MIP5 $5_{\mathrm{HPA}}$, could be responsible for the imprinting effect that was observed. The phenomenon was explained by the variations in the internal pore system structures and the role of mesopores. Marć and co-workers [36] concluded that the imprinting process changed the surface morphological properties, increasing the availability of the porous structure for analyte (higher availability of particles, defined as mesopores). A high level of available mesopores was a preferable factor for sorption materials because mesopores are responsible for a particular surface area of the high affinity adsorption sites.

Subsequently, to confirm the composition of the polymer matrix, the EDS analysis was employed. This analytical technique allowed for the identification of the elemental composition of the material. Here, MIP5 $_{\text {HPA }}$ was analysed, since the nitrogen atoms derived only from the functional monomer of (5), should be easily detected. The EDS spectrum of MIP5 $5_{\text {HPA }}$ is presented in Fig. 5. Nitrogen atoms were

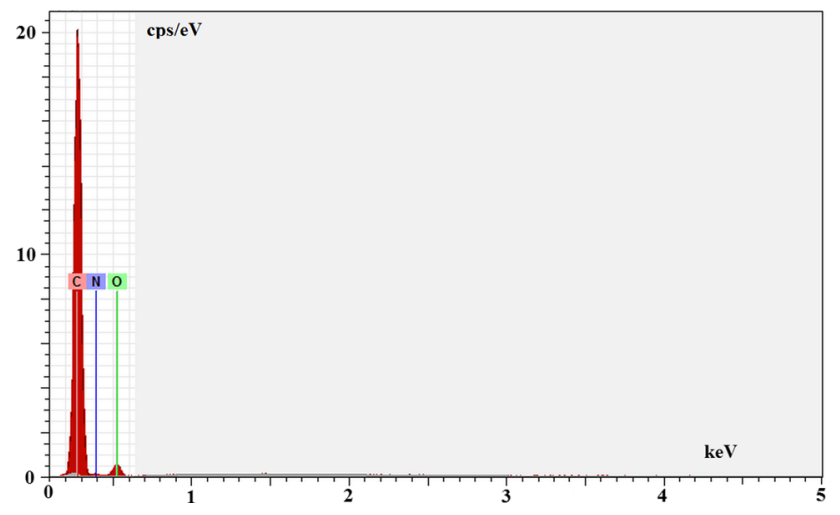

Figure 5 EDS spectrum of $\mathrm{MIP}_{\mathrm{HPA}}$. detected in MIP5 $_{\mathrm{HPA}}$ in the region of $0.35 \mathrm{keV}$. Moreover, the quantitative analysis of MIP5 ${ }_{\mathrm{HPA}}$ estimated the percentage weight of nitrogen, carbon and oxygen. The results were as follows: $\mathrm{N}, 6.80 \pm 0.70 \%$ wt., $\mathrm{C}, 83.27 \pm 0.69 \%$ wt. and $\mathrm{O}, 9.93 \pm 0.58 \%$ wt. The EDS analysis is evidence of the presence of the residues of (5) in the polymer matrix of $\mathrm{MIP}_{\mathrm{HPA}}$.

A critical moment during the synthesis of MIPs relates to effective template removal. This process resulted in the formation of spatial geometries on the surface of MIPs that could be responsible for the specificity of the material. However, taking into account the EDS analysis, it is difficult to prove that the template was efficiently washed out from the polymer matrix, since the HPA molecule does not possess any other heterocyclic atoms, other than the oxygen atom. Here, the fluorescence spectroscopy could be a valuable technique for analysing various acidic metabolites of amino acids, such as 4-hydroxyphenyl lactic acid, HPA and 3,4-hydroxyphenylpropionic acid [37]. The eluates that passed through the MIP $_{\text {HPA }}$ sorbent after extraction (standard extraction in the Soxhlet apparatus without any further steps) and following the subsequent washing sequence with $1 \%$ aq. ammonium hydroxide in methanol and methanol were analysed. Figure 6 presents the three-dimensional fluorescence spectra of fractions.

As can be seen, the template removal step, carried out in the Soxhlet apparatus, had limited effectiveness. The fraction of eluates that passed through MIP5 $_{\text {HPA }}$ after extraction revealed the presence of leaking HPA. The detectable fluorescence was observed in the regions of $\lambda_{\mathrm{em}}=280 \mathrm{~nm}$ and $\lambda_{\mathrm{em}}$ $=570 \mathrm{~nm}$ (Fig. 6a, b). The subsequent washing sequence resulted in a significant reduction in the HPA leakage, decreasing fluorescence intensities (Fig. 6c, d). Thus, the fluorescence spectroscopy could be a valuable method in proving the completeness of the template removal step.

Next, the FT-IR analysis was employed to confirm the structure of $M I P 5_{\mathrm{HPA}}$ and NIP5. Figure 7 presents the spectra.

The spectra of MIP5 $5_{\mathrm{HPA}}$ and NIP5 have a very similar pattern. The characteristic vibration peaks, derived from structural fragments of MIP5 $5_{\mathrm{HPA}}$, could be assigned as follows: at $3454 \mathrm{~cm}^{-1}$ (broad), the $-\mathrm{OH}$ stretching vibrations; at 2991 and at $2959 \mathrm{~cm}^{-1}$, the stretching vibration of the $\mathrm{sp}^{2}$ and $\mathrm{sp}^{3}$ bonds of the $\mathrm{CH}$ from the EGDMA; at $1730 \mathrm{~cm}^{-1}$, the $-\mathrm{C}=\mathrm{O}$ 


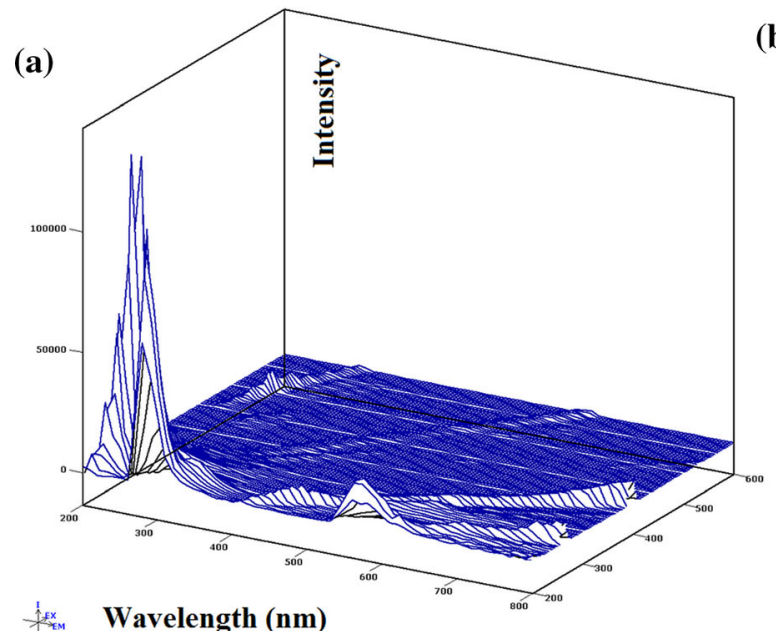

(b)

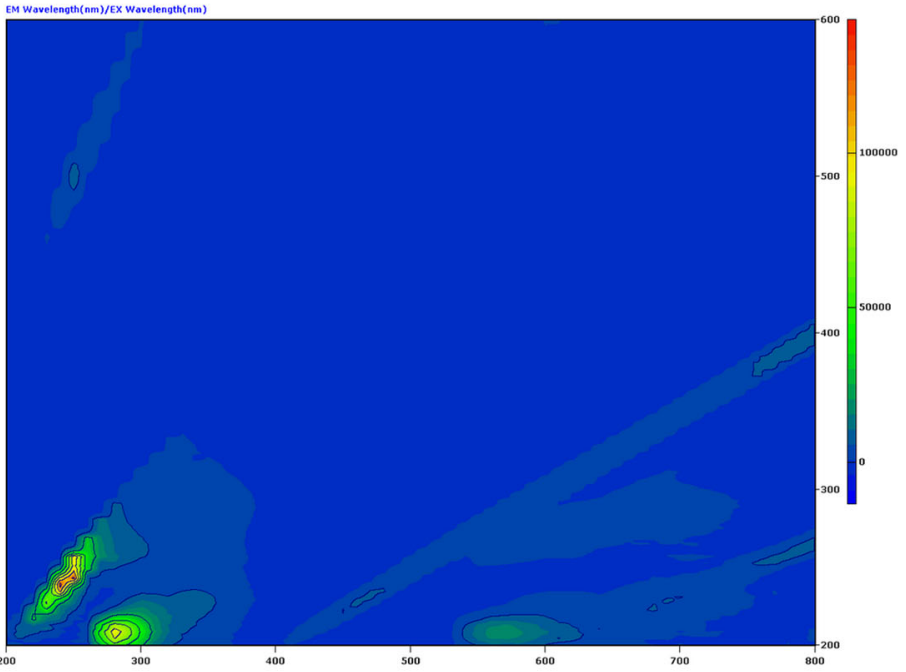

(c)

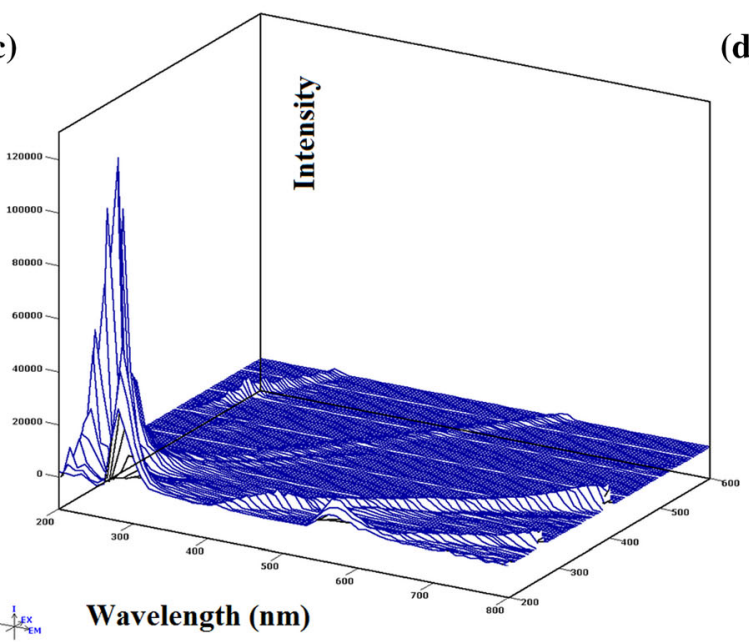

(d)

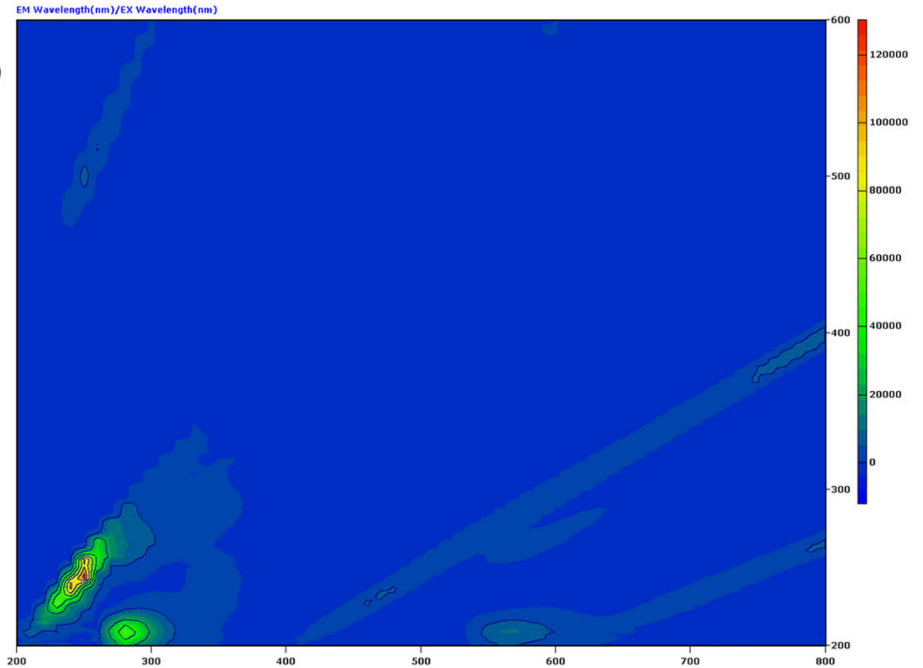

Figure 6 Fluorescence spectra of fraction, passed through MIP5 $5_{\mathrm{HPA}}$ during the template removal: following Soxhlet extraction $(\mathbf{a}, \mathbf{b})$ and the subsequent washing sequence $(\mathbf{c}, \mathbf{d})$.

Figure 7 FT-IR spectra of $\mathrm{MIP}_{\mathrm{HPA}}$ and NIP5.

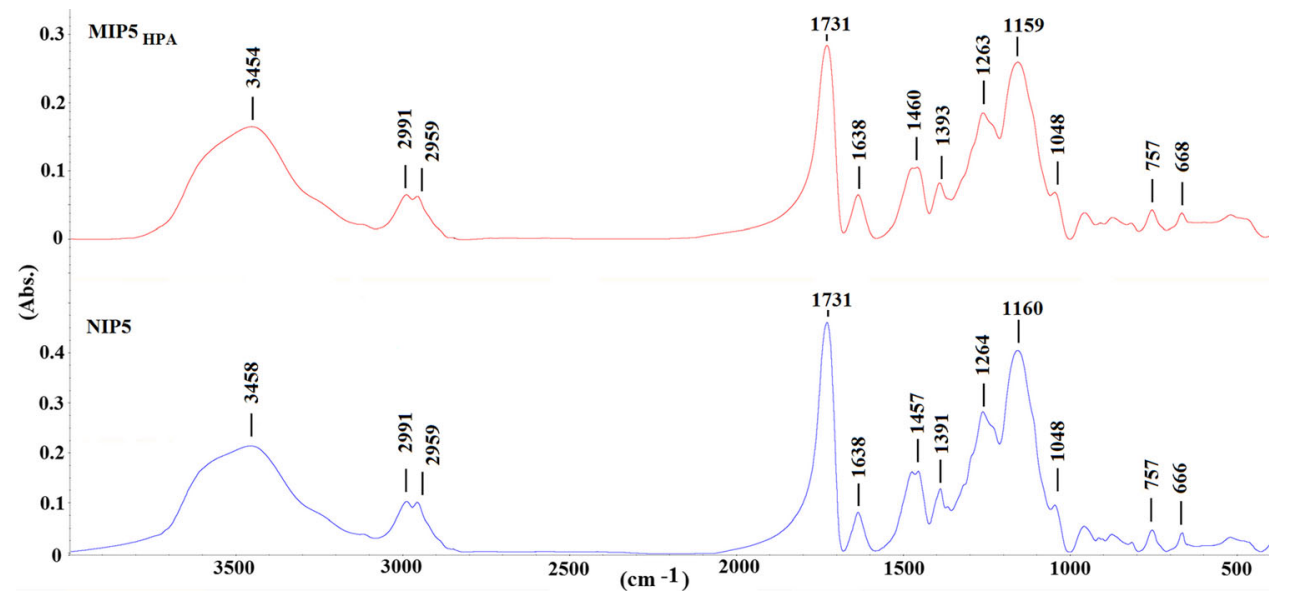

stretching vibration; at $1638 \mathrm{~cm}^{-1}$, the $-\mathrm{C}=\mathrm{C}$ or $-\mathrm{C}=\mathrm{N}$ stretching vibrations from the aromatic imidazole ring; at $1460 \mathrm{~cm}^{-1}$, the asymmetric $-\mathrm{CH}$ deformations; at $1393 \mathrm{~cm}^{-1}$, the $-\mathrm{C}=\mathrm{N}$ stretching modes from 
the ring; at $1263 \mathrm{~cm}^{-1}$ and $1159 \mathrm{~cm}^{-1}$, the $\mathrm{C}-\mathrm{O}-\mathrm{C}$ stretching vibrations at $1048 \mathrm{~cm}^{-1}$, contribute to the $\mathrm{C}-\mathrm{C}-\mathrm{C}$ bending vibrations of the backbone chain; at $757 \mathrm{~cm}^{-1}$, the stretching vibration of the $-\mathrm{C}=\mathrm{N}$ bond which connects the imidazole ring to the vinyl moiety and the $\mathrm{C}-\mathrm{C}-\mathrm{C}$ bending vibrations of the backbone of the aliphatic chain, and at $668 \mathrm{~cm}^{-1}$, the puckering vibration of the imidazole ring [38, 39].

Finally, the TGA analyses of MIP5 $5_{\mathrm{HPA}}$ and NIP5 were carried out to observe the degradation of materials as a function of temperature. The TGA curves are shown in Fig. 8.
As shown, the decomposition patterns of $\mathrm{MIP}_{\mathrm{HPA}}$ and NIP5 are very similar (Fig. 8). The process started at around $200{ }^{\circ} \mathrm{C}$ and continued up to $470{ }^{\circ} \mathrm{C}$. The decomposition step consisted of two stages with the first maximum of weight loss at $310.6{ }^{\circ} \mathrm{C}$ and $310.0{ }^{\circ} \mathrm{C}$ for MIP5 $5_{\text {HPA }}$ and NIP5, respectively, with the loss of nearly $40 \%$ of the total mass. The second maximum was observed at $399.6{ }^{\circ} \mathrm{C}$ and at $398.4{ }^{\circ} \mathrm{C}$ for MIP5 $_{\text {HPA }}$ and NIP5, respectively, with an almost entire loss of the initial mass of material. We could suppose that the initial decomposition was attributed to the short chain degradation, as well as the decarboxylation process, which was also responsible for
Figure 8 TGA curves of $\mathrm{MIP5}_{\mathrm{HPA}}$ (a) and NIP5 (b). Green line represents the loss of weight as a function of temperature; blue line is a derivative of weight loss as a function of temperature.
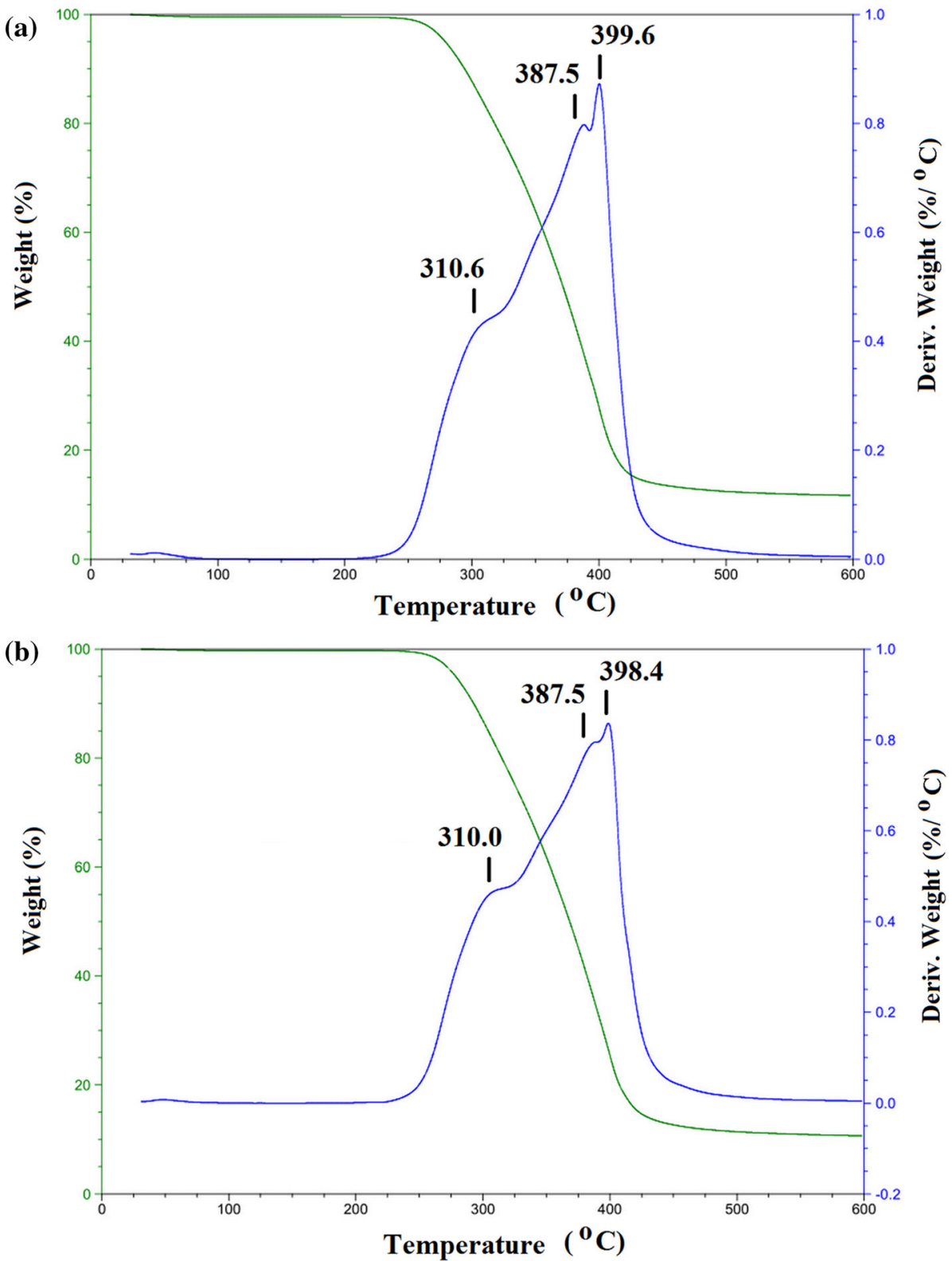
stable decomposition in the range of $300-470{ }^{\circ} \mathrm{C}$. The short maximum of weight loss at $50{ }^{\circ} \mathrm{C}$ could be explained by the loss of intrinsically bound water.

To sum up, the physicochemical characterization proved the presence of monomer residues in the polymer matrix, and confirmed the different sorption capability of MIP5 $5_{\mathrm{HPA}}$ and NIP5.

\section{Binding properties and selectivity}

In the next step, the binding properties of MIP5 $5_{\text {HPA }}$ and NIP5 in relation to the NHPA were evaluated. Equilibrium adsorption studies were carried out and the data obtained were inserted into two mathematical models, viz. Langmuir and Freundlich.

Firstly, the Langmuir model, transformed to the Scatchard equation, Eq. 4, was examined. The system, which fits well with the model gives a straight line on the Scatchard plot, with a slope equalling -(1/ $K_{\mathrm{d}}$ ) and a $y$-intercept equalling $B_{\max } / K_{\mathrm{d}}$. The system which has more than one population of adsorption sites is characterized by more than one line. The Scatchard plots for MIP5 ${ }_{\mathrm{HPA}}$ and NIP5 are shown in Fig. 9a. The analysis revealed two straight lines for MIP5 $_{\text {HPA }}$ and only one line for NIP5. This means that two classes of adsorption sites for NHPA were predominant in MIP5 $5_{\mathbf{H P A}}$, with two $K_{\mathrm{d}}$ and two $B_{\max }$ values: $K_{\mathrm{d}} \quad(1)=30.2 \mu \mathrm{g} \quad \mathrm{L}^{-1}$ and $B_{\max }$ (1) $=153.7 \mu \mathrm{g} \mathrm{g}^{-1}$ for the higher affinity adsorption sites, and $K_{\mathrm{d}} \quad(2)=357 \mu \mathrm{g} \quad \mathrm{L}^{-1}$ and $B_{\max }$ (2) $=416 \mu \mathrm{g} \mathrm{g}^{-1}$ for the lower affinity adsorption sites. Thus, MIP5 $5_{\text {HPA }}$ possessed specific adsorption sites for NHPA. By contrast, NIP5 had only one low affinity adsorption site, characterized by the value of $K_{\mathrm{d}}=2 \mathrm{mg} \mathrm{L}^{-1}$ and $B_{\max }=0.9 \mathrm{mg} \mathrm{g}^{-1}$.

Next, the Freundlich model, represented by Eq. 5, was used to confirm the heterogeneity of the adsorption sites. That model fits well with the adsorption data in the low concentration regions. The straight lines of $\log B$ versus $\log F$ with the regression coefficient of $r^{2}=0.945$ and 0.968 for MIP5 ${ }_{\mathrm{HPA}}$ and NIP5, respectively, provided evidence that adsorption could be described by the Freundlich equation (Fig. 9b). The estimated values of $m$ for MIP5 ${ }_{\mathrm{HPA}}$ and NIP5 were 0.39 and 0.91 , respectively. The results indicated that MIP5 $5_{\mathrm{HPA}}$ had a more heterogeneous population of adsorption sites with respect to NIP5 (the heterogeneity increased as the value of $m$ decreased).

Finally, the selectivity of MIP5 $5_{\mathrm{HPA}}$ and NIP5 in relation to the target analyte, NHPA and the group of structurally related compounds were investigated. Six analogues were analysed, viz. PHE, HPA (acting as the template), NBA, NFE, 3CT and 3NT. The noncompetitive stationary binding experiments were carried out, the $B$ were calculated according to Eq. 1, and the $K_{\mathrm{d}}$ and AF according to Eq. 2 and Eq. 3, respectively. The results are shown in Fig. 10.

As shown, the $B$ on MIP5 $5_{\text {HPA }}$ strongly depend on the physicochemical nature of the analysed compound. Both tested amino-acids, 3-NT and 3-CT, were only slightly adsorbed $(B=0.007$ and $\left.0.168 \mu \mathrm{mol} \mathrm{g}^{-1}\right)$. This could be explained by the presence of the electron-rich amine group, as well as the steric hindrance of bulky amino-acid function. A higher binding capacity was observed for NFE $\left(B=1.896 \mu \mathrm{mol} \mathrm{g}^{-1}\right)$, the compound that possesses two functional groups, electron-withdrawing $-\mathrm{NO}_{2}$ and electron donating $-\mathrm{OH}$, substituted to the aromatic ring, which can act as a hydrogen bond donoracceptor, capable of interacting with (5) residues in the polymer network. Significantly higher $B$ values were noted for all acidic compounds, viz. PHE, NHPA and HPA $(B=2.316,2.361$ and $2.365 \mu \mathrm{mol} \mathrm{g}^{-1}$ ). It should be emphasized that HPA was used as the template molecule in the synthesis, and NHPA was the target analyte. All those compounds are aliphatic carboxylic acids that possess the
Figure 9 Scatchard plots a and Freundlich isotherms b for MIP5 $5_{\mathrm{HPA}}$ and NIP5.
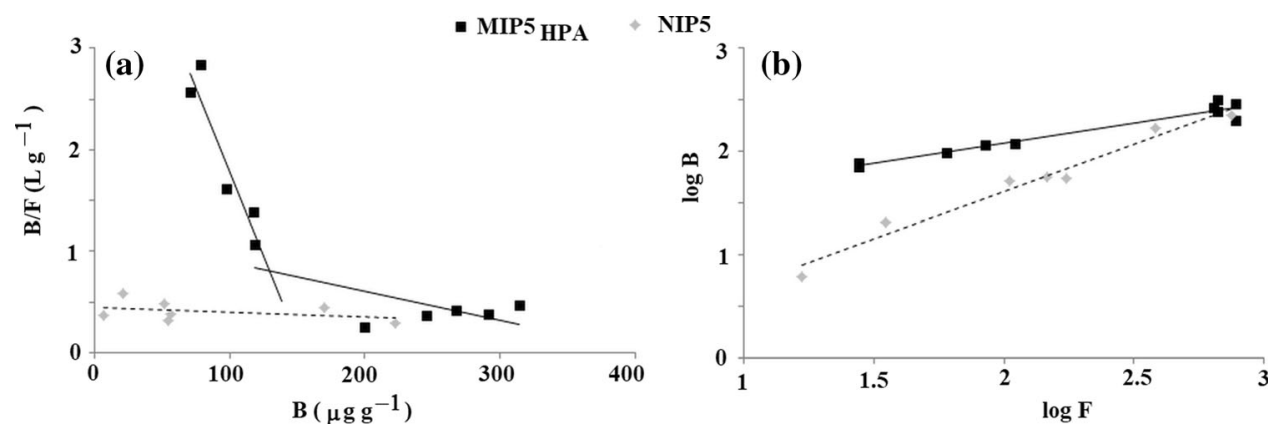
Figure $10 B$ and AF of NHPA and its structural analogues on MIP5 $_{\mathrm{HPA}}$ and NIP5 (c $=7.5 \mu \mathrm{mol} \mathrm{L}-1, n=3$, RSD $<7.1 \%)$.

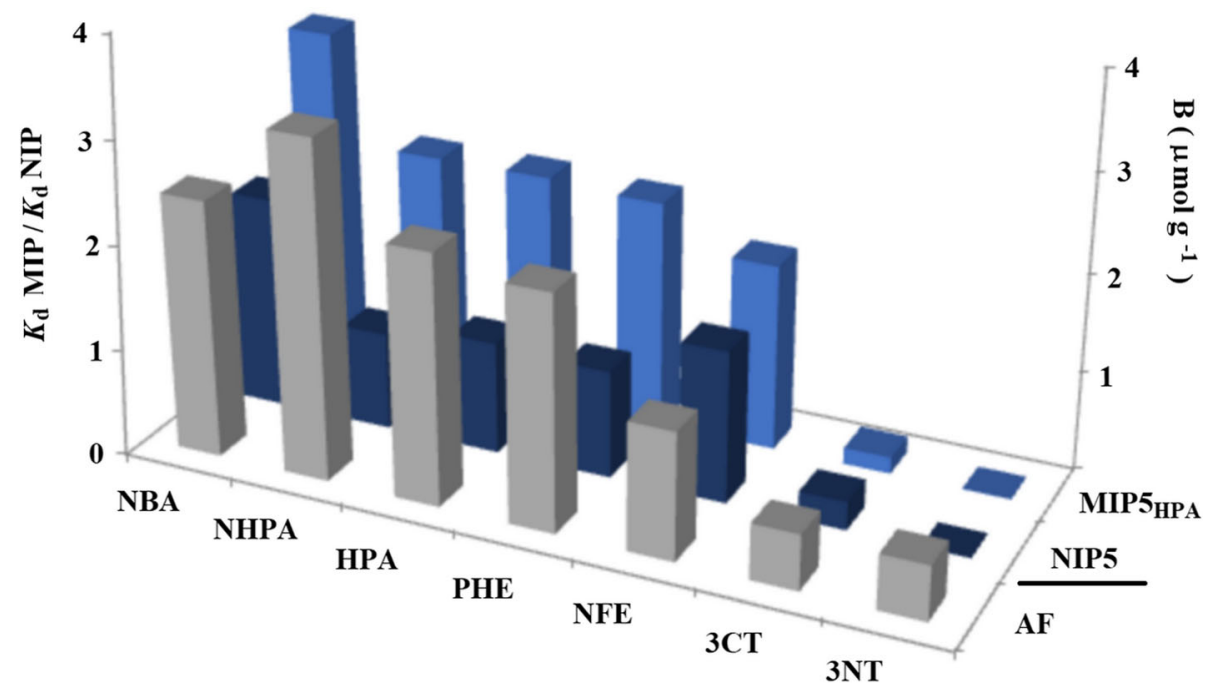

Theoretical explanation of selectivity

$-\mathrm{COOH}$ group, linked by the methylene linker with the aromatic ring. The carboxylic group could play a crucial role in the adsorption process, either in neutral or ionizable forms (the adsorption process was carried out in aqueous solution). Nevertheless, the increase in $B$ between those carboxylic compounds were observed as follows: PHE $<$ NHPA $<$ HPA. Thus, it could be deduced that the presence of substituted functions in the aromatic ring also governed the adsorption. The highest $B=3.450 \mu \mathrm{mol} \mathrm{g}^{-1}$ was noted for NBA. This compound is an aromatic carboxylic acid, which is characterized by stronger acidity. The resonance effect and the presence of an electron withdrawing $-\mathrm{NO}_{2}$ group in meta position, can stabilize ionizable forms of compound. This could explain the high affinity of NBA to MIP $5_{\mathrm{HPA}}$. The $B$ of tested compounds on NIP5 did not follow the same trend, emphasizing the specificity of MIP5 $5_{\text {HPA }}$. Low $B$ of PHE, NHPA and HPA on NIP5 and high $B$ on $\mathrm{MIP5}_{\mathrm{HPA}}$ resulted in high specificity $(\mathrm{AF}=2.39,2.54$ and 3.43, respectively).

To sum up, it should be stated that the $B$ of the template HPA and the analyte NHPA were very similar in terms of MIP5 $_{\text {HPA }}$ but varied in relation to NIP5, emphasizing the specificity of the imprinted material in relation to NHPA. However, the highest $B$ were noted for NBA, affecting the selectivity of MIP5 $_{\text {HPA }}$. To obtain an insight into the molecular basis of the specificity and selectivity of MIP5 $5_{\mathrm{HPA}}$, a theoretical analysis of the interactions between the selected analytes and the polymer, together with an evaluation of binding energies in the in silico generated cavities, were carried out.
The theoretical simulations of the adsorption process of different analytes, viz. NHPA, HPA, PHE, NBA, NFE, 3CT and $3 \mathrm{NT}$ on MIP5 $5_{\mathrm{HPA}}$ were performed to assess the selectivity of the MIP5 $5_{\text {HPA }}$ cavity. The interactions of the NHPA in the cavity of MIP5 $5_{\text {HPA, }}$ have been previously discussed.

Therefore, firstly the template molecule of the HPA was analysed (Fig. 11a). This created three interactions with the polymeric chain during adsorption: one $\pi-\pi \mathrm{T}$-shaped interaction with the imidazole ring, one non-classical hydrogen interaction between the $\mathrm{O}$ atom from the $-\mathrm{COOH}$ group and the $\mathrm{H}$ atom from the imidazole ring of the polymeric chain, and one hydrophobic $\pi$-alkyl type interaction with the $-\mathrm{CH}_{3}$ group of the EGDMA residue. However, considering adsorption conditions and the $\mathrm{pH}$ dependent equilibrium, the interactions between the ionizable forms were also considered. Here, one additional electrostatic interaction between the $-\mathrm{COO}^{-}$group and the imidazolium cation from the polymeric chain, and one additional non-classical hydrogen bond were observed. Different types of interactions between the analyte and the solvent were also present in the analysed system: three classical and three non-classical hydrogen bonds between the $\mathrm{H}$ or $\mathrm{O}$ atoms from the $-\mathrm{COOH}$ or $-\mathrm{OH}$ groups of the HPA and the methanol molecules.

Next, the PHE adsorption on the MIP5 $5_{\text {HPA }}$ chain was evaluated. Here, no interactions between the analyte and the polymer were observed. However, given the possibility of ionization of appropriate 


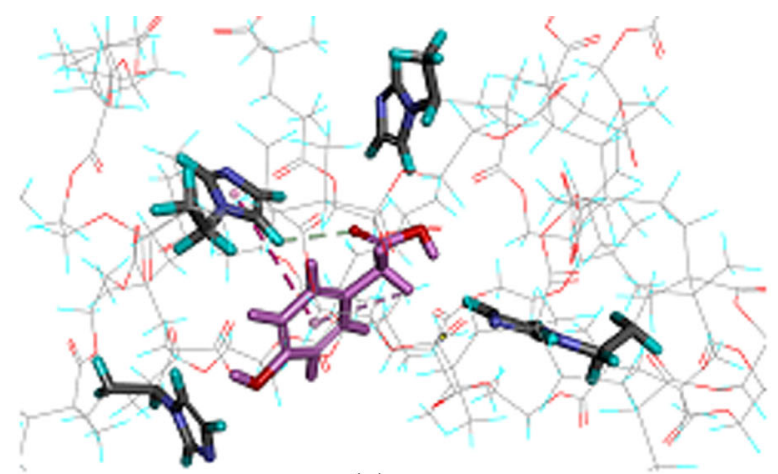

(a)

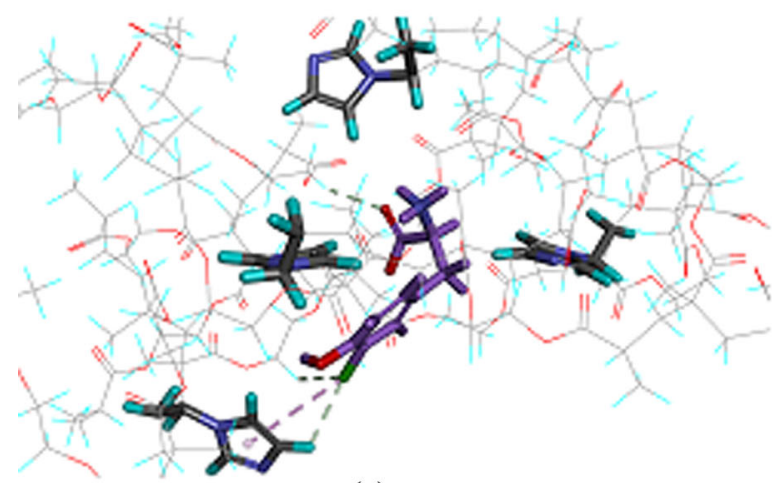

(c)

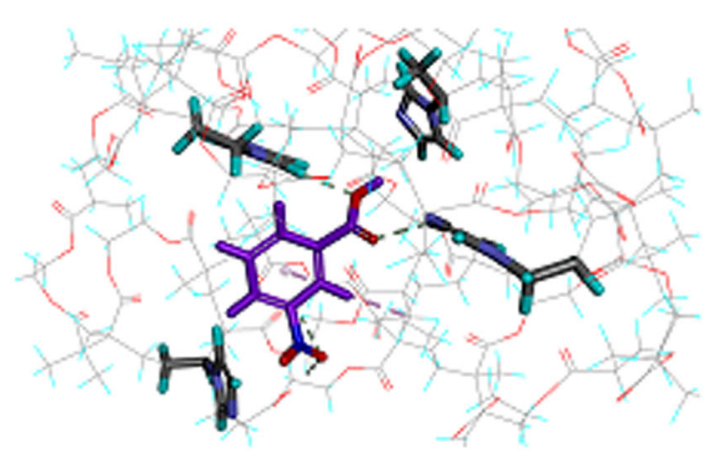

(b)

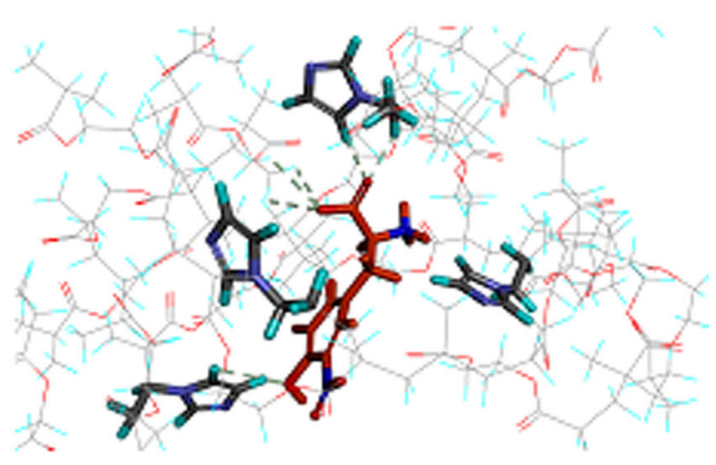

(d)

Figure 11 A view of the analytes of HPA (a), NBA (b), 3CT (c) and $3 \mathrm{NT}$ (d) in the theoretically generated cavity of MIP5 $5_{\mathrm{HPA}}$.

functional groups in the analysed system, the electrostatic interaction between the $-\mathrm{COO}^{-}$anion from the analyte and the imidazolium cation from the chain, as well as that of the hydrogen bond between the $\mathrm{O}$ atom from the $-\mathrm{COO}^{-}$group of the PHE and the $\mathrm{H}$ atom from the aromatic ring of the monomer residue were observed. Two non-classical hydrogen bonds were observed between the $\mathrm{COOH}$ group or aromatic ring of the PHE and the methanol molecules.

In the case of the NBA, four non-classical hydrogen bonds between the $\mathrm{O}$ atoms from the $-\mathrm{COOH}$ or the $\mathrm{NO}_{2}$ groups, and the $\mathrm{H}$ atoms from the imidazole rings or the $-\mathrm{CH}_{2}-$ groups of the EGDMA residues in the polymeric chain, were noted (Fig. 11b). Additionally, one $\pi$-alkyl type interaction was observed between the analyte and the $-\mathrm{CH}_{3}$ group of the EGDMA residue. Once again, taking into account the possibility of ion creation in the analysed system, three additional electrostatic interactions between the NBA and the chain were observed. Moreover, numerous interactions of the NBA with the solvent were observed. Three classical hydrogen bonds were formed with the participation of the $\mathrm{O}$ atoms from the $-\mathrm{COOH}$ and $-\mathrm{NO}_{2}$ groups of the NBA and water, or methanol molecules. Five non-classical hydrogen bonds were created between the $\mathrm{O}$ atoms or the aromatic ring of the analyte and the $\mathrm{H}$ atoms of the water or methanol, and one $\pi$-lone pair-type interaction was formed between the aromatic ring of the NBA and the methanol.

During the theoretical simulation of the NFE adsorption process on MIP5 $5_{\mathrm{HPA}}$, three non-classical hydrogen bonds, formed between the $\mathrm{O}$ atoms from the $-\mathrm{NO}_{2}$ group of the NFE and the $\mathrm{H}$ atoms from the imidazole ring or the $-\mathrm{CH}_{2}-$ group of the EGDMA residue, were noted. Two hydrophobic $\pi$-alkyl-type interactions were also formed between the aromatic ring of the NFE and the $-\mathrm{CH}_{3}$ groups of the EGDMA residue, and one $\pi$-lone pair type interaction was created between the aromatic ring of the NFE and the $\mathrm{O}$ atom of the EGDMA residue. Considering the possibility of creation of the imidazolium cation in the polymeric chain, additional electrostatic interaction was formed between the cation and the $-\mathrm{NO}_{2}$ group of the NFE. The analyte interacted with the solvent, creating four classical and four non-classical hydrogen bonds between the $\mathrm{O}$ or $\mathrm{H}$ atoms of the - 
$\mathrm{OH}$ and $-\mathrm{NO}_{2}$ groups and the methanol or water molecules, and one $\pi$-sigma-type interaction between the aromatic ring of the NFE and the methanol molecule.

The analysis of $3 \mathrm{CT}$ adsorption on $\mathrm{MIP}_{\mathrm{HPA}}$ provided information relating to three non-classical hydrogen bonds, which were found between the chlorine atom or the $\mathrm{O}$ atom from the $-\mathrm{COO}^{-}$group of the analyte, and the $\mathrm{H}$ atoms from the monomer residue ring or the $-\mathrm{CH}_{2}-$ groups of the EGDMA residues (Fig. 11c). One interaction between the chlorine atom and the aromatic ring of the imidazole was also observed. Considering the possibility of imidazolium cation creation, no additional interactions of 3CT with the chain were observed. Here, numerous interactions of the analyte with solvent were formed: five classical and three non-classical hydrogen bonds between the $\mathrm{H}$ atoms from the $\mathrm{NH}_{3}{ }^{+}$group or the $\mathrm{O}$ atoms from the $-\mathrm{OH}$ or $-\mathrm{COO}^{-}$ groups of the 3CT and methanol molecules.

Finally, the analyte of 3NT created six non-classical hydrogen bonds with a polymeric matrix between the $\mathrm{O}$ atoms from the $-\mathrm{COO}^{-}$or $-\mathrm{OH}$ groups and the $\mathrm{H}$ atoms from the aromatic ring or alkyl chain of monomer residues, or $\mathrm{H}$ atoms from the $-\mathrm{CH}_{2}-$ groups of the EGDMA residue (Fig. 11d). Similar to the $3 \mathrm{CT}$ adsorption simulation on $\mathrm{MIP}_{\mathrm{HPA}}$, the creation of imidazolium ions in the polymeric chain, did not produce additional interactions. The analyte interacted with the solvent, creating three classical and six non-classical hydrogen bonds, with the participation of the $\mathrm{H}$ atoms from the $-\mathrm{NH}_{3}{ }^{+}$group, or the $\mathrm{O}$ atoms from the $-\mathrm{COO}^{-}$or $-\mathrm{NO}_{2}$ groups and the methanol molecules.

It could be concluded that the number of electrostatic interactions correlated well with the experimental $B$ values. The highest $B$ of the NBA $\left(3.450 \mu \mathrm{g} \mathrm{g}^{-1}\right)$ in relation to MIP5 $5_{\mathbf{H P A}}$ are associated with three strong electrostatic interactions with the polymer residues, and the lowest $B$ for $3 \mathrm{CT}$ and $3 \mathrm{NT}$ correlate with no electrostatic interactions. However, it could be also concluded that the interactions of the analyte with the solvent molecules impacted negatively on the selectivity. The highest selectivity was noted for the NHPA, the system with no interactions with solvent molecules. Additionally, mutual interactions with the monomer and cross-linker residues enhanced selectivity. By contrast, the lower selectivity was noted in the case of the NBA due to the presence of classical and non-classical hydrogen bonds, as well as the $\pi$-lone interactions with solvent molecules.

\section{Optimization of solid phase extraction}

The optimization of the SPE protocol is crucial for the characterization of recognition ability of MIPs. The appropriate solvents, used in the washing and eluting steps of the SPE procedure, should favour the development of interactions between the analyte and the monomer residues present in the cavities, but exclude the non-specific adsorption on the surface of the polymer outside the cavities. Therefore, the effect of washing and eluting solvents was investigated in this area. The following solvents were tested: water adjusted to $\mathrm{pH} 4$, hexane, $15 \%$ methanol in water, $15 \%$ methanol in water adjusted to $\mathrm{pH} 4$ and $15 \%$ methanol in water with $1 \%$ of ammonium hydroxide as washing solvents and t-butyl-methyl ether, ethyl acetate and methanol as eluting solvents. The results are presented in Table 1.

As shown, the most significant recovery was noted after applying water, adjusted to $\mathrm{pH} 4$, or hexane as the washing solvent. The greatest recovery, obtained after washing with water, adjusted to $\mathrm{pH} 4$, could be explained by the similar conditions when comparing the loading step, minimizing non-specific adsorption. The recovery obtained after washing with hexane could be explained by the fact that aprotic and nonpolar hexane could reduce the non-specific adsorption in the most effective way. All tested methanolwater washing systems resulted in lower recoveries. This means that the desorption of specifically adsorbed NHPA also occurred. The highly basic, methanol-water washing system, adjusted by $1 \%$ ammonium hydroxide, resulted in an almost total desorption of NHPA and the lowest recovery. The elution solvent was characterized by strong desorption capabilities. Here, methanol was characterized by the highest NHPA desorption percentage, probably due to its polar and protic properties.

To sum up, the impact of the washing solvent was significant for the recovery of the NHPA. Nevertheless, to simplify the separation step and shorten the time of the analysis, the following SPE procedure was selected as the most efficient: loading with $1 \mathrm{~mL}$ of human urine (adjusted to $\mathrm{pH} 4$ ), washing with $1 \mathrm{~mL}$ of water, adjusted to $\mathrm{pH} 4$, followed by $1 \mathrm{~mL}$ of methanol elution. 


\section{Analysis of 4-hydroxyphenylacetic acid conversion}

Finally, the in vitro analysis of the conversion of HPA to NHPA in human urine was carried out to ascertain whether the analysis conditions could affect the levels of NHPA in the nitrate-rich acidified human urine sample. This analysis could also prove the applicability of the newly designed imprinted sorbent. For that purpose, the nitrate and nitrite salts were added to the sample of human urine, spiked with HPA, and adjusted to $\mathrm{pH}$ 2. The optimized SPE protocol was applied and the elution fraction was analysed by the UPLC-MS/MS system. The chromatogram of the human urine sample before the addition of nitrate and nitrite salts, the 4-hydroxyphenylacetic acid, adjusted to $\mathrm{pH} 2$ and the chromatogram of the elution fraction after the test, are presented in Fig. 12.

As shown, the NHPA was detected in the human urine sample (peak in $6.37 \mathrm{~min}$.) with a concentration of $357 \pm 85 \mathrm{ng} \mathrm{L}^{-1}(n=3)$. The elution fraction after the SPE protocol on the MIP5 $5_{\mathrm{HPA}}$ of the human urine sample, spiked with nitrate and nitrite salts and the HPA adjusted to $\mathrm{pH} \mathrm{2,} \mathrm{revealed} \mathrm{an} \mathrm{almost} \mathrm{twofold}$ increase of NHPA to $775 \pm 81 \mathrm{ng} \mathrm{L^{-1 }}(n=3)$. The results could be explained by the conversion of HPA to NHPA in the presence of nitrate and nitrite salts, under the acidic conditions of analysis. Thus, the results could prove that the conditions of SPE could affect the concentration of the NHPA in the sample.

\section{Conclusions}

Among 20 various imprinted polymers, obtained during the design process, the polymer synthesized from 1-vinylimidazole in the presence of 4-hydroxyphenylacetic acid, revealed the highest specificity in relation to 4-hydroxy-3-nitrophenylacetic acid, a metabolite of 3-nitrotyrosine. The theoretical analysis revealed that numerous interactions between the template and monomer, supported by additional interaction with the cross-linker and the solvent, stabilized the prepolymerization complex. The physicochemical characterization proved the presence of monomer residues in the polymer matrix and confirmed the different sorption capability between the imprinted and non-imprinted polymers. The binding capacities of the template molecule of the
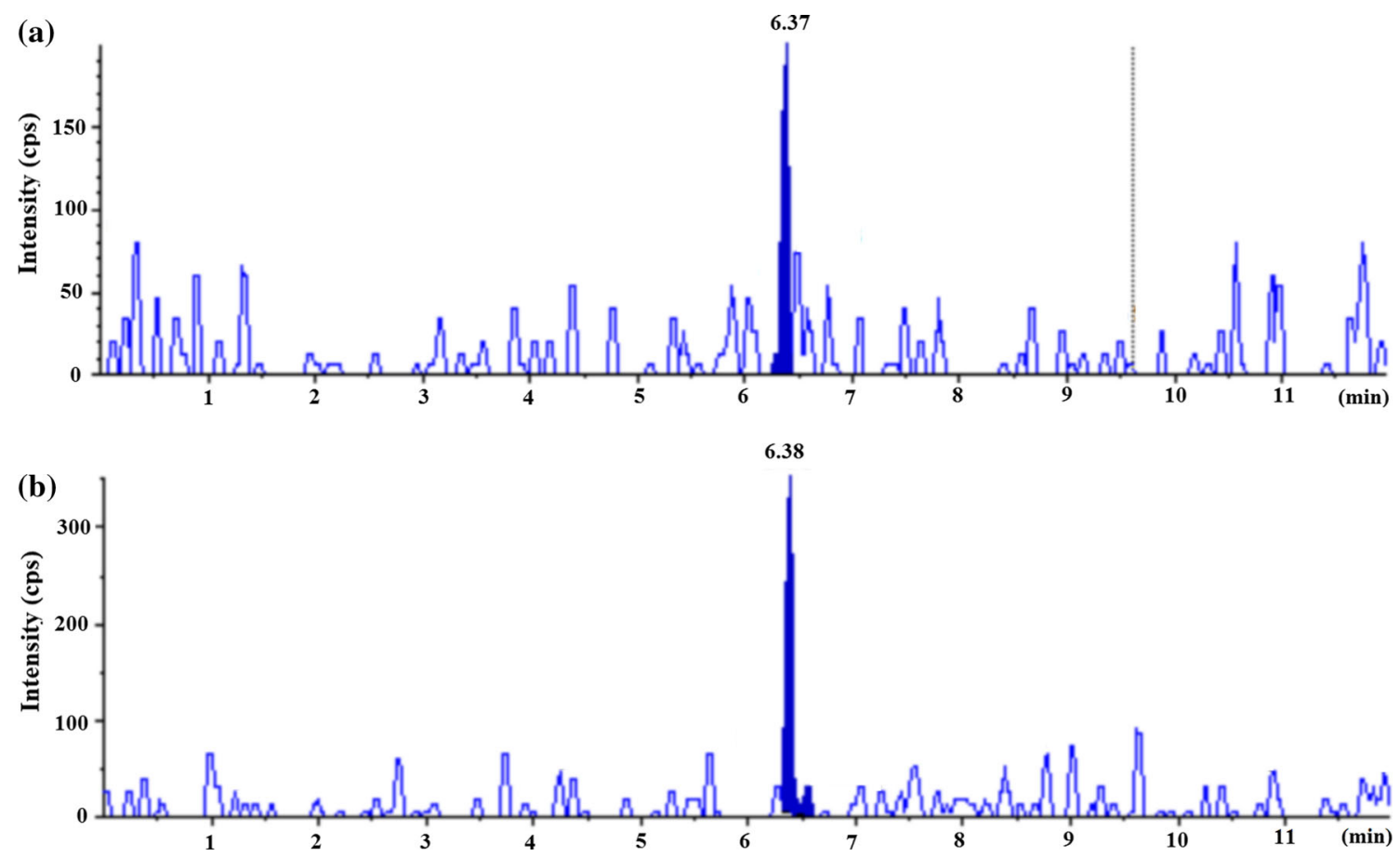

Figure 12 Chromatogram of human urine sample before addition of nitrate and nitrite salts, 4-hydroxyphenylacetic acid and adjustment to $\mathrm{pH} 2$ a and chromatogram of elution fraction, after
SPE protocol of human urine sample, with added nitrate and nitrite salts, spiked with HPA and adjusted to $\mathrm{pH} 2 \mathbf{b}$. 
4-hydroxyphenylacetic acid and the analyte of the 4-hydroxyphenylacetic acid, were very similar on the imprinted polymer, but varied significantly when compared to the binding capacities on the non-imprinted polymer, emphasizing the specificity of the imprinted material. The highest binding capacity, noted for 3-nitrobenzoic acid, was explained by the theoretical analysis, revealing that electrostatic interaction, together with the lack of interactions of the analyte with the solvents, supports selectivity. The optimized solid phase extraction protocol allowed for the analysis of the conversion of the 4-hydroxyphenylacetic acid to 4-hydroxy-3-nitrophenylacetic acid, in the presence of nitrate and nitrite salts, under the acidic conditions of analysis. The results proved that the conditions of the solid phase extraction process could affect the concentration of the 4-hydroxy-3-nitrophenylacetic acid in the sample.

\section{Acknowledgements}

The results presented in this paper were obtained using the resources of the Interdisciplinary Centre for Mathematical and Computational Modelling (ICM) at the University of Warsaw, Poland (G68-2). The LCMS analyses were carried out with the use of the CePT infrastructure financed by the European Union, the European Regional Development Fund within the Operational Program "Innovative economy" for 2007-2013.

\section{Compliance with ethical standards}

Conflict of interest There are no conflicts to declare.

Supplementary information: The online version of this article (https://doi.org/10.1007/s10853-021-058 47-9)

Open Access This article is licensed under a Creative Commons Attribution 4.0 International License, which permits use, sharing, adaptation, distribution and reproduction in any medium or format, as long as you give appropriate credit to the original author(s) and the source, provide a link to the Creative Commons licence, and indicate if changes were made. The images or other third party material in this article are included in the article's Creative Commons licence, unless indicated otherwise in a credit line to the material. If material is not included in the article's Creative Commons licence and your intended use is not permitted by statutory regulation or exceeds the permitted use, you will need to obtain permission directly from the copyright holder. To view a copy of this licence, visit http://creativecommons.org/licen ses/by/4.0/.

\section{References}

[1] BelBruno JJ (2019) Molecularly imprinted polymers. Chem Rev 119:94-119

[2] Crapnell RD, Dempsey-Hibbert NC, Peeters M, Tridente A, Banks CE (2020) Molecularly imprinted polymer based electrochemical biosensors: overcoming the challenges of detecting vital biomarkers and speeding up diagnosis. Talanta Open 2:100018

[3] Sobiech M, Bujak P, Luliński P, Pron A (2019) Semiconductor nanocrystal-polymer hybrid nanomaterials and their application in molecular imprinting. Nanoscale 11:12030-12074

[4] Wackerlig J, Lieberzeit PA (2015) Molecularly imprinted polymer nanoparticles in chemical sensing - Synthesis, characterisation and application. Sens Actuators B Chem 207:144-157

[5] Alexander C, Davidson L, Hayes W (2003) Imprinted polymers: artificial molecular recognition materials with applications in synthesis and catalysis. Tetrahedron 59:2025-2057

[6] Muratsugu S, Shirai S, Tada M (2020) Recent progress in molecularly imprinted approach for catalysis. Tetrahedron Lett $61: 151603$

[7] He S, Zhang L, Bai S, Yang H, Cui Z, Zhang X, Li Y (2021) Advances of molecularly imprinted polymers (MIP) and the application in drug delivery. Eur Polym J 143:110179

[8] Tuwahatu CA, Yeung CC, Lam YW, Vellaisamy Roy VAL (2018) The molecularly imprinted polymer essentials: curation of anticancer, ophthalmic, and projected gene therapy drug delivery systems. J Control Release 287:24-34

[9] Luliński P (2017) Molecularly imprinted polymers based drug delivery devices: a way to application in modern pharmacotherapy - a review. Mater Sci Eng C 76:1344-1353

[10] Luliński P, Giebułtowicz J, Wroczyński P, Maciejewska D (2015) A highly selective molecularly imprinted sorbent for extraction of 2-aminothiazoline-4-carboxylic acid - Synthesis, characterization and application in post-mortem whole blood analysis. J Chromatogr A 1420:16-25

[11] Luliński P, Dana M, Maciejewska D (2014) Synthesis and characterization of 4-(2-aminoethyl) aniline imprinted 
polymer as a highly effective sorbent of dopamine. Talanta 119:623-631

[12] Luliński P, Maciejewska D (2012) Effective separation of dopamine from bananas on 2-(3,4-dimethoxyphenyl) ethylamine imprinted polymer. J Sep Sci 35:1050-1057

[13] Luliński P, Bamburowicz-Klimkowska M, Dana M, Szutowski M, Maciejewska D (2016) Efficient strategy for the selective determination of dopamine in human urine by molecularly imprinted solid-phase extraction. J Sep Sci 39:895-903

[14] Cowen T, Karim K, Piletsky S (2016) Computational approaches in the design of synthetic receptors - a review. Anal Chim Acta 936:62-74

[15] Sobiech M, Żołek T, Luliński P, Maciejewska D (2014) A computational exploration of imprinted polymer affinity based on voriconazole metabolites. Analyst 139:1779-1788

[16] Bandookwala M, Thakkar D, Sengupta P (2020) Advancement in the analytical quantification of nitroxidative stress biomarker 3-nitrotyrosine in biological matrices. Crit Rev Anal Chem 50:265-289

[17] Jin J, Wang C, Tao Y, Tan Y, Yang D, Gu Y, Deng H, Bai Y, Lu H, Wan Y, Lu Z, Li Y (2011) Determination of 3-nitrotyrosine in human urine samples by surface plasmon resonance immunoassay. Sens Actuators B 153:164-169

[18] Mani AR, Pannala AS, Orie NN, Ollosson R, Harry D, RiceEvans CA, Moore KP (2003) Nitration of endogenous parahydroxyphenylacetic acid and the metabolism of nitrotyrosine. Biochem J 374:521-527

[19] Hoehn T, Janssen S, Mani AR, Brauers G, Moore KP, Schadewaldt P, Mayatepek E (2008) Urinary extraction of the nitrotyrosine metabolite 3-nitro-4-hydroxyphenylacetic acid in preterm and term infants. Neonatology 93:73-76

[20] Ohshima H, Friesen M, Brouet I, Bartsc H (1990) Nitrotyrosine as a new marker for endogenous nitrosation and nitration of proteins. Food Chem Toxicol 28:647-652

[21] Crow JP (1999) Measurement and significance of free and protein-bound 3-nitrotyrosine, 3-chlorotyrosine, and free 3-nitro-4-hydroxyphenylacetic acid in biologic samples: a high-performance liquid chromatography method using electrochemical detection. Methods Enzymol 310:151-160

[22] Chao M-R, Hsu Y-W, Liu H-H, Lin J-H, Hu C-W (2015) Simultaneous detection of 3-nitrotyrosyne and 3-nitro-4hydroxyphenylacetic acid in human urine by online SPE LCMS/MS and their association with oxidative and methylated DNA lesions. Chem Res Toxicol 28:997-1006

[23] Pannala AS, Mani AR, Rice-Evans CA, Moore KP (2006) $\mathrm{pH}-$ dependent nitration of para-hydroxyphenylacetic acid in the stomach. Free Radic Biol Med 41:896-901

[24] Sobiech M, Luliński P, Halik P, Maciejewska D (2017) The selective response of a templated polymer for the cationic drug pentamidine: implications from molecular simulations and experimental data. RSC Adv 7:46881-46893

[25] Dassault Systèmes BIOVIA (2018) Discovery studio modeling environment, release 2019. Dassault Systèmes, San Diego

[26] Frish MJ, Trucks GW, Schlegel HB, Scuseria GE, Robb MA, Cheeseman JR, Zakrzewski VG, Montgomery JAJ, Stratmann RE, Burant JC, Dapprich S, Millam JM, Daniels AD, Kudin KN, Strain MC, Farkas O, Tomasi J, Barone V, Cossi M, Cammi R, Mennucci B, Pomelli C, Adamo C, Clifford S, Ochterski J, Petersson GA, Ayala PY, Cui Q, Morokuma K, Malick DK, Rabuck AD, Raghavachari K, Foresman JB, Cioslowski J, Ortiz JV, Baboul AG, Stefanov BB, Liu G, Liashenko A, Piskorz P, Komaromi I, Gomperts R, Martin RL, Fox DJ, Keith T, Al-Laham MA, Peng CY, Nanayakkara A, Gonzalez C, Challacombe MP, Gill PMW, Johnson B, Chen W, Wong MW, Andres JL, Gonzalez C, Head-Gordon M, Replogle ES, Pople JA (2009) Gaussian 09. Gaussian Inc., Pittsburgh, PA

[27] Brooks BR, Brooks CL, MacKerell AD, Nilsson L, Petrella RJ, Roux B, Won Y, Archontis G, Bartels C, Boresch S, Caflisch A, Caves L, Cui Q, Dinner AR, Feig M, Fischer S, Gao J, Hodoscek M, Im W, Kuczera K, Lazaridis T, Ma J, Ovchinnikov V, Paci R, Pastor RW, Post CB, Pu JZ, Schaefer M, Tidor B, Venable RM, Woodcock HL, Wu X, Yang W, York DM, Karplus M (2009) CHARMM: the biomolecular simulation program. J Comput Chem $30: 1545-1614$

[28] Martínez L, Andrade R, Birgin EG, Martínez JM (2009) Packmol: a package for building initial configurations for molecular dynamics simulations. J Comput Chem 30:2157-2164

[29] Ryckaert JP, Ciccotti G, Berendsen HJC (1977) Numerical integration of the cartesian equations of motion of a system with constraints: molecular dynamics of $n$-alkanes. J Comput Phys 23:327-341

[30] Baggiani C, Giovannoli C, Anfossi L, Passini C, Baravalle P, Giraudi G (2012) A connection between the binding properties of imprinted and non-imprinted polymers: a change of perspective in molecular imprinting. J Am Chem Soc 134:1513-1518

[31] Brunauer S, Emmett PH, Teller E (1938) Adsorption of gases in multimolecular layers. J Am Chem Soc 60:309-319

[32] Sing KSW (1982) Reporting physisorption data for gas/solid systems with special reference to the determination of surface area and porosity. Pure Appl Chem 54:2201-2218

[33] Silvestre-Albero AM, Juarez-Galan JM, Silvestre-Albero J, Rodríguez-Reinoso F (2012) Low-pressure hysteresis in adsorption: an artifact? J Phys Chem C 116:16652-16655 
[34] Barrett EP, Joyner LG, Halenda PP (1951) The determination of pore volume and area distributions in porous substances. I. Computations from nitrogen isotherms. J Am Chem Soc 73:373-380

[35] Jura G, Harkins WD (1946) Surfaces of solids. XIV. a unitary thermodynamic theory of the adsorption of vapors on solids and of insoluble films on liquid subphases. J Am Chem Soc 68:1941-1952

[36] Marć M, Panuszko A, Namieśnik J, Wieczorek PP (2018) Preparation and characterization of dummy-template molecularly imprinted polymers as potential sorbents for the recognition of selected polybrominated diphenyl ethers. Anal Chim Acta 1030:77-95

[37] Yang Y, Liu F, Wan Y (2017) Simultaneous determination of 4-hydroxyphenyl lactic acid, 4-hydroxyphenyl acetic acid, and 3,4-hydroxyphenyl propionic acid in human urine by ultra-high performance liquid chromatography with fluorescence detection. J Sep Sci 40:2117-2122

[38] dos Santos QO, Bezerra MA, de Fátima LG, Diniz KM, Segatelli MG, Germiniano TO, da Silva SV, Tarley CRT (2014) Synthesis, characterization and application of ion imprinted poly(vinylimidazole) for zinc ion extraction/preconcentration with FAAS determination. Quim Nova 37:63-68

[39] Talu M, Demiroglu EU, Yurdakul S, Badoglu S (2015) FTIR, Raman and NMR spectroscopic and DFT theoretical studies on poly (N-vinylimidazole). Spectrochim Acta A 134:267-275

Publisher's Note Springer Nature remains neutral with regard to jurisdictional claims in published maps and institutional affiliations. 